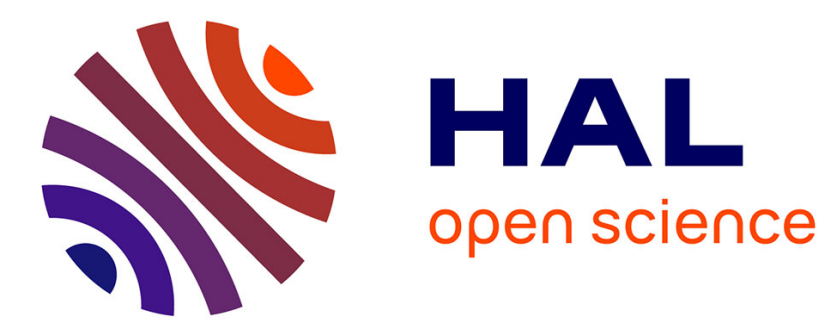

\title{
Approximation of mono-dimensional hyperbolic systems: a lattice Boltzmann scheme as a relaxation method
}

Benjamin Graille

\section{To cite this version:}

Benjamin Graille. Approximation of mono-dimensional hyperbolic systems: a lattice Boltzmann scheme as a relaxation method. Journal of Computational Physics, 2014, 266, pp.74-88. 10.1016/j.jcp.2014.02.017 . hal-00919835

\section{HAL Id: hal-00919835 \\ https://hal.science/hal-00919835}

Submitted on 17 Dec 2013

HAL is a multi-disciplinary open access archive for the deposit and dissemination of scientific research documents, whether they are published or not. The documents may come from teaching and research institutions in France or abroad, or from public or private research centers.
L'archive ouverte pluridisciplinaire HAL, est destinée au dépôt et à la diffusion de documents scientifiques de niveau recherche, publiés ou non, émanant des établissements d'enseignement et de recherche français ou étrangers, des laboratoires publics ou privés.

\section{(ㅇ)(1) $\$$}

Distributed under a Creative Commons Attribution - NonCommercial - NoDerivatives $\mid 4.0$ 


\title{
Approximation of mono-dimensional hyperbolic systems: a lattice Boltzmann scheme as a relaxation method
}

\author{
B. Graille ${ }^{\mathrm{a}}$ \\ ${ }^{a}$ Univ. Paris-Sud, Laboratoire de Mathématiques, UMR 8628, Orsay, F-91405, France, CNRS, Orsay, F-91405
}

\begin{abstract}
We focus on mono-dimensional hyperbolic systems approximated by a particular lattice Boltzmann scheme. The scheme is described in the framework of the multiple relaxation times method and stability conditions are given. An analysis is done to link the scheme with an explicit finite differences approximation of the relaxation method proposed by Jin and Xin. Several numerical illustrations are given for the transport equation, the Burgers equation, the $p$-system, and the full compressible Euler system.
\end{abstract}

Keywords: lattice Boltzmann scheme, relaxation method, hyperbolic system

2010 MSC: 35L60, 65M06, 65M12

\section{Introduction}

The strength of the lattice Boltzmann schemes lies in their effectivity. They are intensively used in academic and industrial contexts for numerical simulations of fluid dynamics. Their links with the mesoscopic physics and in particular with the Boltzmann equation make that these schemes are especially well adapted to simulate fluid phenomenas obtained by asymptotic limits from kinetic theory. However, it is sometimes awkward to fix the several parameters of a lattice Boltzmann scheme in order to simulate a given equation, even if this equation is written into a conservative form: the conservation of the energy is classically a difficulty that can involve to use two different schemes coupled by a source term [1]. Other very particular schemes were proposed and investigated in order to simulate the full compressible Euler system, with substantial works on the equilibria $[2,3,4,5,6]$.

In this contribution, a new lattice Boltzmann scheme is introduced in order to approximate any mono-dimensional hyperbolic system. The followed methodology is to treat separately the equations of the system by forgiving the Boltzmann equation as much as possible. Usually, in order to increase the dimension of the system - that is the number of conservation equationsdensities with larger velocities are introduced with two consequences: first, the lattice of the velocities is extended with the obvious difficulties concerning the boundary conditions; second, added new velocities deeply modifies the scheme so that all previous investigations have to be redone. The proposed scheme denoted by $\mathrm{D}_{1} \mathrm{Q}_{2}^{n}$ is built by duplicating for each of the $n$ conserved moments the well-known and simplest lattice Boltzmann scheme: the $D_{1} Q_{2}$ (one spatial

Email address: Benjamin.Graille@math.u-psud.fr (B. Graille)

Preprint submitted to Journal of Computational Physics

September 30, 2013 
dimension and two discrete velocities). Therefore, the results on the scalar equation can easily be extended to the system of $n$ equations. Moreover, as the boundary conditions are written on the densities in the framework of the lattice Boltzmann schemes, the decoupling of the density functions extremely simplifies the choices of the incoming densities on the bounds to fit the boundary conditions on the moments.

In [7], Jin and Xin introduced the relaxation method to replace a non linear hyperbolic system of dimension $n$ by a linear hyperbolic system of dimension $2 n$ with a stiff source term-called a relaxation term as it enforces the added moment to relax to the flux of the initial system. The convergence of this method when the relaxation term becomes dominant was investigated in $[8,9]$. Many publications deal then with numerical relaxation schemes [10, 11, 12]. In [13], Junk reinterprets besides the lattice Boltzmann method-in particular the $\mathrm{D}_{2} \mathrm{Q}_{9}$ - as an explicit finite differences discretization of a relaxation formulation for the incompressible Navier-Stokes equation in the diffusion scaling. In this paper, the proposed $D_{1} Q_{2}^{n}$ scheme is related to a particular discretization of the relaxation method: a splitting between the linear hyperbolic part treated with an explicit finite differences discretization (Lax-Friedrichs discretization) and the relaxation part treated with an explicit Euler solver.

The first section of this paper is devoted to the scalar case: the $D_{1} Q_{2}$ scheme is written into the framework of d'Humières [14]; the equivalent equations are given up to the second order by using the Taylor expansion method [15, 16]; the description of the scheme as a discretization of the relaxation method is then done and stability conditions are given; finally numerical illustrations for the transport equation and for the Burger's equation are performed. In the second section, we consider the case of $n$-dimensional hyperbolic systems: the $\mathrm{D}_{1} \mathrm{Q}_{2}^{n}$ scheme is introduced and described; the Taylor expansion method is then used to obtain the second order equivalent equations and the link with the discretization of the relaxation method is done; finally numerical illustrations for the $p$-system and for the full compressible Euler equation are performed.

\section{The $D_{1} Q_{2}$ scheme for the $1-D$ scalar equation}

In this section, we consider the following mono-dimensional hyperbolic equation

$$
\partial_{t} \mathrm{u}(t, x)+\partial_{x} \varphi(\mathrm{u})(t, x)=0, \quad t>0, x \in \mathbb{R},
$$

where the flux $\varphi$ is a smooth function on $\mathbb{R}$. A two-velocities lattice Boltzmann scheme is used to approximate the solution of this equation.

\subsection{Description of the scheme}

We use the notation proposed by d'Humières in [14] by considering $\mathcal{L}$, a regular lattice in one dimension of space with typical mesh size $\Delta x$. The time step $\Delta t$ is determined after the specification of the velocity scale $\lambda$ by the relation:

$$
\Delta t=\frac{\Delta x}{\lambda} .
$$

For the scheme denoted by $D_{1} Q_{2}$, we introduce $v=(-\lambda, \lambda)$ the set of the two velocities and we assume that for each node $x$ of $\mathcal{L}$, and each $v_{j}$ in $v$, the point $x+v_{j} \Delta t$ is also a node of the lattice $\mathcal{L}$. The aim of the $\mathrm{D}_{1} \mathrm{Q}_{2}$ scheme is to compute a particles distribution vector $\boldsymbol{f}=\left(f_{0}, f_{1}\right)^{\mathrm{T}}$ 
on the lattice $\mathcal{L}$ at discrete values of time: it is a numerical scheme to approximately solve the PDEs

$$
\partial_{t} f_{j}+v_{j} \cdot \nabla f_{j}=-\frac{1}{\tau_{j}}\left(f_{j}-f_{j}^{\mathrm{eq}}\right), \quad 0 \leq j \leq 1,
$$

on a grid in space and time where $f_{j}^{\text {eq }}$ describes the distribution $f_{j}$ at the equilibrium and $\tau_{j}$ is the relaxation time (related on $f_{j}$ ). The scheme splits into two phases for each time iteration: first, the relaxation phase that is local in space, and second, the transport phase for which an exact characteristic method is used.

The framework proposed by d'Humières [14] reduced here to the two moments denoted by $\boldsymbol{m}=(\mathrm{u}, \mathrm{v})^{\mathrm{T}}$ and defined for each space point $x \in \mathcal{L}$ and for each time $t$ by

$$
\mathrm{u}=f_{0}+f_{1}, \quad \mathrm{v}=\lambda\left(-f_{0}+f_{1}\right) .
$$

The matrix of the moments $\boldsymbol{M}$ such that $\boldsymbol{m}=\boldsymbol{M} \boldsymbol{f}$ satisfies

$$
\boldsymbol{M}=\left(\begin{array}{cc}
1 & 1 \\
-\lambda & \lambda
\end{array}\right), \quad \boldsymbol{M}^{-1}=\left(\begin{array}{cc}
\frac{1}{2} & -\frac{1}{2 \lambda} \\
\frac{1}{2} & \frac{1}{2 \lambda}
\end{array}\right)
$$

Let us now describe one time step of the scheme. The start point is the density vector $f(x, t)$ in $x \in \mathcal{L}$ at time $t$, the moments are computed by

$$
\boldsymbol{m}(x, t)=\boldsymbol{M} \boldsymbol{f}(x, t) .
$$

The relaxation phase then reads

$$
\mathrm{u}^{\star}(x, t)=\mathrm{u}(x, t), \quad \mathrm{v}^{\star}(x, t)=\mathrm{v}(x, t)+s\left(\mathrm{v}^{\mathrm{eq}}(x, t)-\mathrm{v}(x, t)\right),
$$

where $s$ is the relaxation parameter and $\mathrm{v}^{\mathrm{eq}}$ the second moment at equilibrium that is a function of $u$. As a consequence, the first moment $u$ is conserved during the relaxation phase. The densities are then computed after the relaxation phase by

$$
f^{\star}(x, t)=M^{-1} \boldsymbol{m}^{\star}(x, t) .
$$

The transport phase finally reads

$$
f_{j}(x, t+\Delta t)=f_{j}^{\star}\left(x-v_{j} \Delta t, t\right), \quad 0 \leq j \leq 1 .
$$

\subsection{Asymptotic Analysis : the Taylor expansion method}

The aim of this section is to perform the equivalent equations of the scheme and in particular to fix the equilibrium value $\mathrm{v}^{\mathrm{eq}}$ as a function of $\mathrm{u}$ in order to ensure that the scheme is consistent with (1). This reasoning consists in a formal development of the distribution functions $f(x, t)$ at small $\Delta t$ and $\Delta x$, assuming that these functions are regular enough to use the Taylor formula. The results of this section are particular cases of the general expansion of Dubois $[15,16]$. The interested reader can find proofs in Appendix A.

Proposition 1.1 (zeroth order). Defining the vectors $\boldsymbol{m}^{\mathrm{eq}}=\left(\mathrm{u}, \mathrm{v}^{\mathrm{eq}}\right)^{\mathrm{T}}$ and $\boldsymbol{f}^{\mathrm{eq}}=\boldsymbol{M}^{-1} \boldsymbol{m}^{\mathrm{eq}}$, we have

$$
f_{j}=f_{j}^{\mathrm{eq}}+O(\Delta t), \quad f_{j}^{\star}=f_{j}^{\mathrm{eq}}+O(\Delta t), \quad 0 \leq j \leq 1 .
$$


Proposition 1.2 (First order macroscopic equation). The first moment $\mathrm{u}$ satisfies the partial differential equation

$$
\partial_{t} \mathrm{u}+\partial_{x} \mathrm{v}^{\mathrm{eq}}=O(\Delta t)
$$

The choice $\mathrm{v}^{\mathrm{eq}}=\varphi(\mathrm{u})$ is then done so that $\mathrm{u}$ satisfies (1) at order 1 .

We then define the equilibrium default $\theta$ by using the particular derivatives $\mathrm{d}_{t}^{j}=\partial_{t}+v_{j} \partial_{x}$, $0 \leq j \leq 1$

$$
\theta=\sum_{j=0}^{1} v_{j} \mathrm{~d}_{t}^{j} f_{j}^{\mathrm{eq}} .
$$

The equilibrium default $\theta$ can then be rewritten into the form

$$
\theta=\partial_{t} \mathrm{v}^{\mathrm{eq}}+\lambda^{2} \partial_{x} \mathrm{u}
$$

Lemma 1.3 (Transition lemma). The second moment $\mathrm{v}$ satisfies

$$
\mathrm{v}=\mathrm{v}^{\mathrm{eq}}-\frac{\Delta t}{s} \theta+O\left(\Delta t^{2}\right), \quad \mathrm{v}^{\star}=\mathrm{v}^{\mathrm{eq}}+\Delta t\left(1-\frac{1}{s}\right) \theta+O\left(\Delta t^{2}\right) .
$$

Moreover, we have

$$
f_{j}^{\star}-f_{j}=\Delta t \mathrm{~d}_{t}^{j} f_{j}^{\mathrm{eq}}+O\left(\Delta t^{2}\right), \quad 0 \leq j \leq 1 .
$$

Proposition 1.4 (Second order macroscopic equation). The first moment $\mathrm{u}$ satisfies the secondorder partial differential equation

$$
\partial_{t} \mathrm{u}+\partial_{x} \varphi(\mathrm{u})=\Delta t \sigma \partial_{x}\left(\left(\lambda^{2}-\left(\varphi^{\prime}(\mathrm{u})\right)^{2}\right) \partial_{x} \mathrm{u}\right)+O\left(\Delta t^{2}\right),
$$

with $\sigma=1 / s-1 / 2$.

Let us remark that this second-order macroscopic equation (13) then contains a diffusion term with a regularization effect if $\sigma>0$ (that is $s<2$ ) and $\left|\varphi^{\prime}(\mathrm{u})\right|<\lambda$. These conditions are indeed compatible with the stability conditions of the section 1.4.

\subsection{Link with the relaxation method}

The relaxation method introduced by Jin and Xin [7] to solve the conservation equation (1) consists in forming a linear hyperbolic system with a stiff source term:

$$
\left\{\begin{array}{l}
\partial_{t} \mathrm{u}^{\epsilon}+\partial_{x} \mathrm{v}^{\epsilon}=0, \\
\partial_{t} \mathrm{v}^{\epsilon}+a \partial_{x} \mathrm{u}^{\epsilon}=-\frac{1}{\epsilon}\left(\mathrm{v}^{\epsilon}-\varphi\left(\mathrm{u}^{\epsilon}\right)\right),
\end{array}\right.
$$

where $\epsilon$ is a small positive parameter. This kind of approximation was proposed in the general setting of the quasilinear systems of hyperbolic conservation laws and possesses some very interesting features. Natalini proves in $[8,9]$ that $\mathrm{u}^{\epsilon}$ and $\mathrm{v}^{\epsilon}$ converge to $\mathrm{u}$ and $\varphi(\mathrm{u})$ when $\epsilon$ goes to zero under some technical assumptions where $u$ is the unique entropy solution in the sense of Kružkov [17]. 
In this section we write the $D_{1} Q_{2}$ scheme as a discretization of the relaxation system. Indeed, denoting $\mathrm{u}_{i}^{n}=\mathrm{u}\left(x_{i}, t^{n}\right), \mathrm{v}_{i}^{n}=\mathrm{v}\left(x_{i}, t^{n}\right), x_{i} \in \mathcal{L}$ and $t^{n}=n \Delta t$, we have

$$
\begin{aligned}
\mathrm{v}_{i}^{n \star} & =\mathrm{v}_{i}^{n}-s\left(\mathrm{v}_{i}^{n}-\varphi\left(\mathrm{u}_{i}^{n}\right)\right), \\
\mathrm{u}_{i}^{n+1} & =\frac{1}{2}\left(\mathrm{u}_{i+1}^{n}+\mathrm{u}_{i-1}^{n}\right)-\frac{\Delta t}{2 \Delta x}\left(\mathrm{v}_{i+1}^{n \star}-\mathrm{v}_{i-1}^{n \star}\right), \\
\mathrm{v}_{i}^{n+1} & =\frac{1}{2}\left(\mathrm{v}_{i+1}^{n \star}+\mathrm{v}_{i-1}^{n \star}\right)-\lambda^{2} \frac{\Delta t}{2 \Delta x}\left(\mathrm{u}_{i+1}^{n}-\mathrm{u}_{i-1}^{n}\right) .
\end{aligned}
$$

We then reinterpret the scheme as a splitting of the relaxation system (14) between the relaxation part (15) and the hyperbolic part $(16,17)$. The relaxation part is treated by the explicit Euler method with $\epsilon=\Delta t / s$, and the hyperbolic part by the Lax-Friedrichs method with $a=\lambda^{2}$.

Moreover, we observe that the transport phase of the lattice Boltzmann scheme corresponds exactly to the hyperbolic part in the base of the eigenvectors. Indeed, written the hyperbolic part of Eq. (14) as

$$
\partial_{t} U+\boldsymbol{A} \partial_{x} U=0, \quad \text { with } \quad \boldsymbol{A}=\left(\begin{array}{cc}
0 & 1 \\
\lambda^{2} & 0
\end{array}\right)
$$

we have

$$
\boldsymbol{M}^{-1} \boldsymbol{A} \boldsymbol{M}=\left(\begin{array}{cc}
-\lambda & 0 \\
0 & \lambda
\end{array}\right)
$$

The $D_{1} Q_{2}$ scheme treats then the hyperbolic part of the relaxation system by an upwind method in the base of the eigenvectors.

\subsection{Stability}

In this section, we are interested in the stability of the $D_{1} Q_{2}$ scheme. We first investigate the $L^{2}$-stability for the linear scheme, that is if $\varphi(u)=c u$ with $c$ a real constant. We then give a property of $L^{\infty}$-stability in the general case but with a more restrictive condition.

In the case where $\varphi(u)=c u, c \in \mathbb{R}$, the amplification matrix of the linear $\mathrm{D}_{1} \mathrm{Q}_{2}$ scheme is given by

$$
G(\Delta x, \xi)=\left(\begin{array}{cc}
\left(1-\frac{s}{2}\left(1+\frac{c}{\lambda}\right)\right) e^{-i \Delta x \xi} & \frac{s}{2}\left(1-\frac{c}{\lambda}\right) e^{-i \Delta x \xi} \\
\frac{s}{2}\left(1+\frac{c}{\lambda}\right) e^{i \Delta x \xi} & \left(1-\frac{s}{2}\left(1-\frac{c}{\lambda}\right)\right) e^{i \Delta x \xi}
\end{array}\right)
$$

Proposition 1.5. The linear $\mathrm{D}_{1} \mathrm{Q}_{2}$ scheme is stable for the $L^{2}$-norm if, and only if, $\lambda \geq|c|$ and $s \in[0,2]$.

Proof. Considering the two discs of Gershgorin of the matrix $G(\Delta x, \xi)$, the condition $|c| \leq \lambda$ and $s \in[0,2]$ immediately implies that the two eigenvalues of $G$ have a modulus smaller than 1 . The reciprocal property is trivially true taking $\xi=0$.

Proposition 1.6 (maximum principle). Let $M$ be a positive constant and $\varphi$ a smooth flux function such that $\left|\varphi^{\prime}(\mathrm{u})\right| \leq K$ for $\mathrm{u}$ in the compact $[0, M]$. Considering the $\mathrm{D}_{1} \mathrm{Q}_{2}$ scheme where

- the initial distribution functions are nonnegative $f_{j}(x, 0) \geq 0$, for $0 \leq j \leq 1, x \in \mathcal{L}$,

- the initial global mass $\mathrm{u}^{\text {tot }}=\sum_{x \in \mathcal{L}}\left(f_{0}+f_{1}\right)$ satisfies $\mathrm{u}^{\text {tot }} \leq M$,

- the relaxation parameter $s$ verifies $s \in[0,1]$,

- the velocity of the scheme is such that $\lambda \geq K$, 
then we have

$$
0 \leq f_{j}\left(x, t^{n}\right) \leq M, \quad \text { for } 0 \leq j \leq 1, x \in \mathcal{L}, n \in \mathbb{N} .
$$

As a consequence, the first moment $\mathrm{u}$ remains bounded and nonnegative.

Proof. As the transport phase (8) just exchanges the data, we prove that $f_{j} \geq 0$ implies $f_{j}^{\star} \geq 0$. The problem being invariant by adding a constant to the flux function $\varphi$, we assume that $\varphi(0)=0$. We have for each discrete point $x \in \mathcal{L}$ and each discrete time $t^{n}=n \Delta t$

$$
\begin{aligned}
f_{0}^{\star} & =\left(1-\frac{s}{2}\right) f_{0}+\frac{s}{2} f_{1}-\frac{s}{2 \lambda} \varphi\left(f_{0}+f_{1}\right), \\
f_{1}^{\star} & =\frac{s}{2} f_{0}+\left(1-\frac{s}{2}\right) f_{1}+\frac{s}{2 \lambda} \varphi\left(f_{0}+f_{1}\right) .
\end{aligned}
$$

Written $\varphi\left(f_{0}+f_{1}\right)=\varphi^{\prime}(\xi)\left(f_{0}+f_{1}\right)$ for one $\xi \in[0, M]$ yields

$$
\begin{aligned}
& f_{0}^{\star}=\left(1-\frac{s}{2}\left(1+\frac{\varphi^{\prime}(\xi)}{\lambda}\right)\right) f_{0}+\frac{s}{2}\left(1-\frac{\varphi^{\prime}(\xi)}{\lambda}\right) f_{1}, \\
& f_{1}^{\star}=\frac{s}{2}\left(1+\frac{\varphi^{\prime}(\xi)}{\lambda}\right) f_{0}+\left(1-\frac{s}{2}\left(1-\frac{\varphi^{\prime}(\xi)}{\lambda}\right)\right) f_{1} .
\end{aligned}
$$

The assumptions $s \in[0,1]$ and $\lambda \geq K$ then immediately imply that $f_{0}^{\star}$ and $f_{1}^{\star}$ are nonnegative linear combinations of $f_{0}$ and $f_{1}$, so that are nonnegative. The superior bound is then a consequence of the conservation of the global first moment $\mathrm{u}^{\text {tot }}$.

Remark 1.7. The assumption $\mathrm{u}^{\text {tot }} \leq M$ can be removed in the case where the flux $\varphi$ is $K$ lipschitzienne over $\mathbb{R}$.

\subsection{Numerical illustrations}

In this section, we perform two numerical simulations, one for the transport equation with a constant velocity, and one for the Burger's equation. The lattice $\mathcal{L}$ is reduced to $[0,1]$ and a homogeneous Neumann condition is added to treat the boundaries. In order to visualize the properties of the $D_{1} Q_{2}$ scheme, the initial condition is chosen of two types: first a smooth function and second a Riemann problem type function.

\subsubsection{The transport equation}

Let $c$ be a real constant, we consider in this section $\varphi(u)=c u$.

In Fig. 1, the left (resp. right) figure shows the initial and the final (at time $T=0.4$ ) moment $\mathrm{u}$ for several relaxation parameters $s$ for smooth initial condition (resp. riemann problem). The number of points in space $N=200$ had been chosen in order to visualize that the maximum principle is fulfilled when $s \in[0,1]$ and is not when $s \in] 1,2]$ (the condition $\lambda \geq|c|$ is true). The Tbl. 1 (resp. Tbl. 2) shows the convergence of the $L^{2}$-norm for several relaxation parameters $s$ when $\Delta x$ goes to zero for smooth initial condition (resp. riemann problem). Each line corresponds to the integer $k \in\{3, \ldots, 16\}$ with $\Delta x=2^{-k}$. We then verify numerically that the scheme is consistent at order 1 with the transport equation in the general case and at order 2 if $s=2$, for smooth solutions. The convergence is lowered when the solution is less regular. 

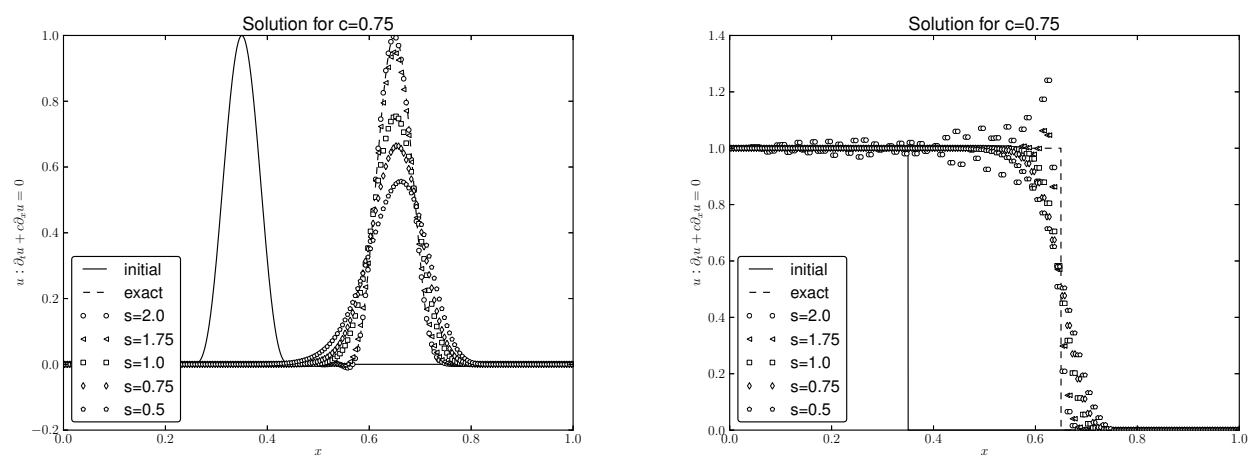

Figure 1: Transport equation with $c=0.75$ at final time $T=0.4$ (left: smooth solution, right: riemann problem)

\begin{tabular}{lcccccc}
\hline$k s$ & 2.000 & 1.900 & 1.750 & 1.000 & 0.750 & 0.500 \\
\hline 3 & $1.536 \mathrm{e}-01$ & $1.416 \mathrm{e}-01$ & $1.256 \mathrm{e}-01$ & $8.104 \mathrm{e}-02$ & $7.881 \mathrm{e}-02$ & $8.113 \mathrm{e}-02$ \\
4 & $1.733 \mathrm{e}-01$ & $1.714 \mathrm{e}-01$ & $1.712 \mathrm{e}-01$ & $2.062 \mathrm{e}-01$ & $2.288 \mathrm{e}-01$ & $2.550 \mathrm{e}-01$ \\
5 & $1.319 \mathrm{e}-01$ & $1.153 \mathrm{e}-01$ & $1.073 \mathrm{e}-01$ & $1.495 \mathrm{e}-01$ & $1.757 \mathrm{e}-01$ & $2.100 \mathrm{e}-01$ \\
6 & $4.897 \mathrm{e}-02$ & $4.697 \mathrm{e}-02$ & $5.138 \mathrm{e}-02$ & $1.145 \mathrm{e}-01$ & $1.405 \mathrm{e}-01$ & $1.719 \mathrm{e}-01$ \\
7 & $1.254 \mathrm{e}-02$ & $1.429 \mathrm{e}-02$ & $2.162 \mathrm{e}-02$ & $7.983 \mathrm{e}-02$ & $1.049 \mathrm{e}-01$ & $1.357 \mathrm{e}-01$ \\
8 & $3.113 \mathrm{e}-03$ & $4.850 \mathrm{e}-03$ & $9.913 \mathrm{e}-03$ & $4.990 \mathrm{e}-02$ & $7.081 \mathrm{e}-02$ & $9.927 \mathrm{e}-02$ \\
9 & $7.761 \mathrm{e}-04$ & $1.991 \mathrm{e}-03$ & $4.836 \mathrm{e}-03$ & $2.863 \mathrm{e}-02$ & $4.329 \mathrm{e}-02$ & $6.599 \mathrm{e}-02$ \\
10 & $1.943 \mathrm{e}-04$ & $9.263 \mathrm{e}-04$ & $2.412 \mathrm{e}-03$ & $1.551 \mathrm{e}-02$ & $2.448 \mathrm{e}-02$ & $3.990 \mathrm{e}-02$ \\
11 & $4.863 \mathrm{e}-05$ & $4.522 \mathrm{e}-04$ & $1.208 \mathrm{e}-03$ & $8.096 \mathrm{e}-03$ & $1.311 \mathrm{e}-02$ & $2.233 \mathrm{e}-02$ \\
12 & $1.216 \mathrm{e}-05$ & $2.241 \mathrm{e}-04$ & $6.041 \mathrm{e}-04$ & $4.138 \mathrm{e}-03$ & $6.794 \mathrm{e}-03$ & $1.188 \mathrm{e}-02$ \\
13 & $3.039 \mathrm{e}-06$ & $1.117 \mathrm{e}-04$ & $3.022 \mathrm{e}-04$ & $2.092 \mathrm{e}-03$ & $3.461 \mathrm{e}-03$ & $6.136 \mathrm{e}-03$ \\
14 & $7.598 \mathrm{e}-07$ & $5.577 \mathrm{e}-05$ & $1.512 \mathrm{e}-04$ & $1.052 \mathrm{e}-03$ & $1.747 \mathrm{e}-03$ & $3.121 \mathrm{e}-03$ \\
15 & $1.900 \mathrm{e}-07$ & $2.787 \mathrm{e}-05$ & $7.559 \mathrm{e}-05$ & $5.277 \mathrm{e}-04$ & $8.778 \mathrm{e}-04$ & $1.574 \mathrm{e}-03$ \\
16 & $4.749 \mathrm{e}-08$ & $1.393 \mathrm{e}-05$ & $3.780 \mathrm{e}-05$ & $2.642 \mathrm{e}-04$ & $4.400 \mathrm{e}-04$ & $7.904 \mathrm{e}-04$ \\
\hline slope & $2.000 \mathrm{e}+00$ & $1.000 \mathrm{e}+00$ & $9.999 \mathrm{e}-01$ & $9.979 \mathrm{e}-01$ & $9.965 \mathrm{e}-01$ & $9.937 \mathrm{e}-01$ \\
\hline
\end{tabular}

Table 1: Transport equation with $c=0.75$ at final time $T=0.4$ (smooth solution: error in $L^{2}$ norm)

\begin{tabular}{lcccccc}
\hline$k s$ & 2.000 & 1.900 & 1.750 & 1.000 & 0.750 & 0.500 \\
\hline 3 & $2.722 \mathrm{e}-01$ & $2.657 \mathrm{e}-01$ & $2.590 \mathrm{e}-01$ & $2.649 \mathrm{e}-01$ & $2.758 \mathrm{e}-01$ & $2.893 \mathrm{e}-01$ \\
4 & $8.353 \mathrm{e}-02$ & $8.611 \mathrm{e}-02$ & $9.415 \mathrm{e}-02$ & $1.696 \mathrm{e}-01$ & $2.027 \mathrm{e}-01$ & $2.389 \mathrm{e}-01$ \\
5 & $1.488 \mathrm{e}-01$ & $1.372 \mathrm{e}-01$ & $1.304 \mathrm{e}-01$ & $1.434 \mathrm{e}-01$ & $1.587 \mathrm{e}-01$ & $1.832 \mathrm{e}-01$ \\
6 & $1.055 \mathrm{e}-01$ & $9.036 \mathrm{e}-02$ & $8.323 \mathrm{e}-02$ & $1.066 \mathrm{e}-01$ & $1.225 \mathrm{e}-01$ & $1.444 \mathrm{e}-01$ \\
7 & $8.651 \mathrm{e}-02$ & $7.416 \mathrm{e}-02$ & $7.188 \mathrm{e}-02$ & $9.591 \mathrm{e}-02$ & $1.082 \mathrm{e}-01$ & $1.251 \mathrm{e}-01$ \\
8 & $6.158 \mathrm{e}-02$ & $4.995 \mathrm{e}-02$ & $5.070 \mathrm{e}-02$ & $7.838 \mathrm{e}-02$ & $8.932 \mathrm{e}-02$ & $1.038 \mathrm{e}-01$ \\
9 & $5.568 \mathrm{e}-02$ & $4.470 \mathrm{e}-02$ & $4.497 \mathrm{e}-02$ & $6.609 \mathrm{e}-02$ & $7.494 \mathrm{e}-02$ & $8.675 \mathrm{e}-02$ \\
10 & $4.421 \mathrm{e}-02$ & $3.434 \mathrm{e}-02$ & $3.570 \mathrm{e}-02$ & $5.515 \mathrm{e}-02$ & $6.270 \mathrm{e}-02$ & $7.268 \mathrm{e}-02$ \\
11 & $3.460 \mathrm{e}-02$ & $2.684 \mathrm{e}-02$ & $2.954 \mathrm{e}-02$ & $4.657 \mathrm{e}-02$ & $5.289 \mathrm{e}-02$ & $6.125 \mathrm{e}-02$ \\
12 & $2.710 \mathrm{e}-02$ & $2.089 \mathrm{e}-02$ & $2.424 \mathrm{e}-02$ & $3.909 \mathrm{e}-02$ & $4.442 \mathrm{e}-02$ & $5.146 \mathrm{e}-02$ \\
13 & $2.230 \mathrm{e}-02$ & $1.732 \mathrm{e}-02$ & $2.043 \mathrm{e}-02$ & $3.288 \mathrm{e}-02$ & $3.735 \mathrm{e}-02$ & $4.326 \mathrm{e}-02$ \\
14 & $1.783 \mathrm{e}-02$ & $1.406 \mathrm{e}-02$ & $1.707 \mathrm{e}-02$ & $2.763 \mathrm{e}-02$ & $3.140 \mathrm{e}-02$ & $3.637 \mathrm{e}-02$ \\
15 & $1.403 \mathrm{e}-02$ & $1.151 \mathrm{e}-02$ & $1.432 \mathrm{e}-02$ & $2.324 \mathrm{e}-02$ & $2.641 \mathrm{e}-02$ & $3.059 \mathrm{e}-02$ \\
16 & $1.111 \mathrm{e}-02$ & $9.517 \mathrm{e}-03$ & $1.202 \mathrm{e}-02$ & $1.954 \mathrm{e}-02$ & $2.220 \mathrm{e}-02$ & $2.572 \mathrm{e}-02$ \\
\hline slope & $3.374 \mathrm{e}-01$ & $2.746 \mathrm{e}-01$ & $2.527 \mathrm{e}-01$ & $2.502 \mathrm{e}-01$ & $2.501 \mathrm{e}-01$ & $2.501 \mathrm{e}-01$ \\
\hline
\end{tabular}

Table 2: Transport equation with $c=0.75$ at final time $T=0.4$ (riemann problem: error in $L^{2}$ norm) 

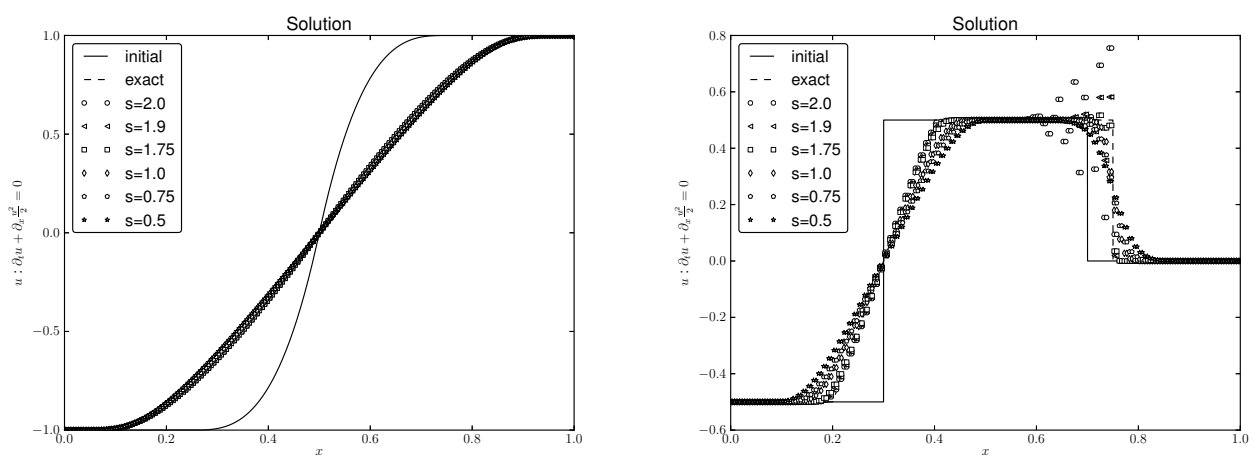

Figure 2: Burger's equation at final time $T=0.2$ (left: smooth solution, right: discontinuous solution)

\begin{tabular}{lcccccc}
\hline$k s$ & 2.000 & 1.900 & 1.750 & 1.000 & 0.750 & 0.500 \\
\hline 3 & $1.378 \mathrm{e}-01$ & $1.378 \mathrm{e}-01$ & $1.378 \mathrm{e}-01$ & $1.378 \mathrm{e}-01$ & $1.378 \mathrm{e}-01$ & $1.378 \mathrm{e}-01$ \\
4 & $4.301 \mathrm{e}-02$ & $4.019 \mathrm{e}-02$ & $3.878 \mathrm{e}-02$ & $7.735 \mathrm{e}-02$ & $1.029 \mathrm{e}-01$ & $1.344 \mathrm{e}-01$ \\
5 & $1.416 \mathrm{e}-02$ & $1.036 \mathrm{e}-02$ & $1.047 \mathrm{e}-02$ & $4.100 \mathrm{e}-02$ & $6.042 \mathrm{e}-02$ & $8.991 \mathrm{e}-02$ \\
6 & $4.256 \mathrm{e}-03$ & $2.441 \mathrm{e}-03$ & $4.038 \mathrm{e}-03$ & $2.142 \mathrm{e}-02$ & $3.334 \mathrm{e}-02$ & $5.389 \mathrm{e}-02$ \\
7 & $1.200 \mathrm{e}-03$ & $8.378 \mathrm{e}-04$ & $1.836 \mathrm{e}-03$ & $1.114 \mathrm{e}-02$ & $1.789 \mathrm{e}-02$ & $3.033 \mathrm{e}-02$ \\
8 & $3.172 \mathrm{e}-04$ & $3.565 \mathrm{e}-04$ & $8.763 \mathrm{e}-04$ & $5.698 \mathrm{e}-03$ & $9.306 \mathrm{e}-03$ & $1.620 \mathrm{e}-02$ \\
9 & $8.073 \mathrm{e}-05$ & $1.655 \mathrm{e}-04$ & $4.274 \mathrm{e}-04$ & $2.878 \mathrm{e}-03$ & $4.743 \mathrm{e}-03$ & $8.381 \mathrm{e}-03$ \\
10 & $2.036 \mathrm{e}-05$ & $7.977 \mathrm{e}-05$ & $2.111 \mathrm{e}-04$ & $1.448 \mathrm{e}-03$ & $2.398 \mathrm{e}-03$ & $4.271 \mathrm{e}-03$ \\
11 & $5.142 \mathrm{e}-06$ & $3.919 \mathrm{e}-05$ & $1.050 \mathrm{e}-04$ & $7.270 \mathrm{e}-04$ & $1.207 \mathrm{e}-03$ & $2.161 \mathrm{e}-03$ \\
12 & $1.289 \mathrm{e}-06$ & $1.942 \mathrm{e}-05$ & $5.238 \mathrm{e}-05$ & $3.644 \mathrm{e}-04$ & $6.061 \mathrm{e}-04$ & $1.087 \mathrm{e}-03$ \\
13 & $3.206 \mathrm{e}-07$ & $9.669 \mathrm{e}-06$ & $2.616 \mathrm{e}-05$ & $1.825 \mathrm{e}-04$ & $3.037 \mathrm{e}-04$ & $5.456 \mathrm{e}-04$ \\
14 & $8.019 \mathrm{e}-08$ & $4.824 \mathrm{e}-06$ & $1.307 \mathrm{e}-05$ & $9.131 \mathrm{e}-05$ & $1.521 \mathrm{e}-04$ & $2.734 \mathrm{e}-04$ \\
15 & $2.017 \mathrm{e}-08$ & $2.410 \mathrm{e}-06$ & $6.534 \mathrm{e}-06$ & $4.568 \mathrm{e}-05$ & $7.610 \mathrm{e}-05$ & $1.369 \mathrm{e}-04$ \\
16 & $5.043 \mathrm{e}-09$ & $1.204 \mathrm{e}-06$ & $3.267 \mathrm{e}-06$ & $2.285 \mathrm{e}-05$ & $3.807 \mathrm{e}-05$ & $6.850 \mathrm{e}-05$ \\
\hline slope & $2.000 \mathrm{e}+00$ & $1.001 \mathrm{e}+00$ & $1.000 \mathrm{e}+00$ & $9.994 \mathrm{e}-01$ & $9.992 \mathrm{e}-01$ & $9.988 \mathrm{e}-01$ \\
\hline
\end{tabular}

Table 3: Burger's equation at final time $T=0.2$ (smooth solution: error in $L^{2}$ norm)

\begin{tabular}{lcccccc}
\hline$k s$ & 2.000 & 1.900 & 1.750 & 1.000 & 0.750 & 0.500 \\
\hline 3 & $2.216 \mathrm{e}-01$ & $2.216 \mathrm{e}-01$ & $2.216 \mathrm{e}-01$ & $2.216 \mathrm{e}-01$ & $2.216 \mathrm{e}-01$ & $2.216 \mathrm{e}-01$ \\
4 & $6.980 \mathrm{e}-02$ & $7.162 \mathrm{e}-02$ & $7.667 \mathrm{e}-02$ & $1.323 \mathrm{e}-01$ & $1.616 \mathrm{e}-01$ & $1.971 \mathrm{e}-01$ \\
5 & $6.115 \mathrm{e}-02$ & $6.021 \mathrm{e}-02$ & $6.318 \mathrm{e}-02$ & $1.031 \mathrm{e}-01$ & $1.246 \mathrm{e}-01$ & $1.555 \mathrm{e}-01$ \\
6 & $6.144 \mathrm{e}-02$ & $5.565 \mathrm{e}-02$ & $5.328 \mathrm{e}-02$ & $7.651 \mathrm{e}-02$ & $9.299 \mathrm{e}-02$ & $1.173 \mathrm{e}-01$ \\
7 & $5.498 \mathrm{e}-02$ & $3.606 \mathrm{e}-02$ & $3.560 \mathrm{e}-02$ & $5.471 \mathrm{e}-02$ & $6.796 \mathrm{e}-02$ & $8.742 \mathrm{e}-02$ \\
8 & $5.584 \mathrm{e}-02$ & $1.553 \mathrm{e}-02$ & $1.163 \mathrm{e}-02$ & $3.619 \mathrm{e}-02$ & $4.732 \mathrm{e}-02$ & $6.312 \mathrm{e}-02$ \\
9 & $8.526 \mathrm{e}-02$ & $1.030 \mathrm{e}-02$ & $1.119 \mathrm{e}-02$ & $2.506 \mathrm{e}-02$ & $3.308 \mathrm{e}-02$ & $4.498 \mathrm{e}-02$ \\
10 & $6.426 \mathrm{e}-02$ & $1.220 \mathrm{e}-02$ & $1.202 \mathrm{e}-02$ & $1.755 \mathrm{e}-02$ & $2.283 \mathrm{e}-02$ & $3.132 \mathrm{e}-02$ \\
11 & $7.534 \mathrm{e}-02$ & $8.772 \mathrm{e}-03$ & $8.165 \mathrm{e}-03$ & $1.192 \mathrm{e}-02$ & $1.545 \mathrm{e}-02$ & $2.130 \mathrm{e}-02$ \\
12 & $6.869 \mathrm{e}-02$ & $3.545 \mathrm{e}-03$ & $2.193 \mathrm{e}-03$ & $7.612 \mathrm{e}-03$ & $1.022 \mathrm{e}-02$ & $1.429 \mathrm{e}-02$ \\
13 & $7.320 \mathrm{e}-02$ & $2.403 \mathrm{e}-03$ & $2.532 \mathrm{e}-03$ & $5.269 \mathrm{e}-03$ & $6.969 \mathrm{e}-03$ & $9.674 \mathrm{e}-03$ \\
14 & $7.377 \mathrm{e}-02$ & $3.012 \mathrm{e}-03$ & $2.930 \mathrm{e}-03$ & $3.808 \mathrm{e}-03$ & $4.852 \mathrm{e}-03$ & $6.611 \mathrm{e}-03$ \\
15 & $7.302 \mathrm{e}-02$ & $2.175 \mathrm{e}-03$ & $2.001 \mathrm{e}-03$ & $2.645 \mathrm{e}-03$ & $3.346 \mathrm{e}-03$ & $4.526 \mathrm{e}-03$ \\
16 & $7.229 \mathrm{e}-02$ & $8.734 \mathrm{e}-04$ & $4.971 \mathrm{e}-04$ & $1.706 \mathrm{e}-03$ & $2.254 \mathrm{e}-03$ & $3.091 \mathrm{e}-03$ \\
\hline slope & $\star \star \star$ & $\star \star \star$ & $\star \star \star$ & $6.324 \mathrm{e}-01$ & $5.700 \mathrm{e}-01$ & $5.500 \mathrm{e}-01$ \\
\hline
\end{tabular}

Table 4: Burger's equation at final time $T=0.2$ (discontinuous solution: error in $L^{2}$ norm) 


\subsubsection{The Burger's equation}

In this section, the flux $\varphi$ is taken to simulate the Burger's equation $\varphi(\mathrm{u})=\mathrm{u}^{2} / 2$.

In Fig. 2, the left (resp. right) figure shows the initial and the final (at time $T=0.2$ ) moment $\mathrm{u}$ for several relaxation parameters $s$ for smooth initial condition (resp. discontinuous initial condition). Concerning the maximum principle, the conditions of Prop. 1.6 are more complicated. The initial data are chosen in order to have $\left|\varphi^{\prime}(\mathrm{u})\right| \leq \lambda$ and we can observe that the principle is fulfilled for $s \in[0,1]$ and is not for $s>1$ with the discontinuous solution.

The smooth initial data has been chosen as a piecewise polynomial function of order three so that an expression of the exact solution can be given. Moreover, this function is increasing so that no shock appears. Its expression reads

$$
\mathrm{u}(x+1 / 2, t=0)= \begin{cases}\operatorname{sign}(x) & \text { for }|x| \geq 1 / 4, \\ \operatorname{sign}(x)\left(1+(4|x|-1)^{3}\right) & \text { for }|x| \leq 1 / 4 .\end{cases}
$$

The discontinuous initial data is a piecewise constant function with two discontinuities: the first one at $x=0.3$ and the second one at $x=0.7$. The left discontinuity leads to a rarefaction wave whereas the right one leads to a shock wave.

The Tbl. 3 (resp. Tbl. 4) shows the convergence of the $L^{2}$-norm for several relaxation parameters when $\Delta x$ goes to zero for smooth initial condition (resp. discontinuous initial condition). We then verify numerically that the scheme is consistent at order 1 with the Burger's equation in the general case and at order 2 if $s=2$, for smooth solutions. In the case of the discontinuous initial condition, we observe a lower convergence if $s \in[0,1]$ but no convergence rate if $s>1$ even if the error seems to be small.

\section{The $D_{1} Q_{2}^{n}$ scheme for the $1-D$ system}

In this section, we consider the following mono-dimensional hyperbolic system

$$
\partial_{t} \mathrm{u}(t, x)+\partial_{x} \varphi(\mathrm{u})(t, x)=0, \quad t>0, x \in \mathbb{R},
$$

where the unknown $\mathrm{u}$ is a vector of $\mathbb{R}^{n}$ and the flux $\varphi$ is a smooth function over $\mathbb{R}^{n}$, for which the jacobian matrix $\mathrm{d} \varphi(\mathrm{u})$ is diagonalizable for each $\mathrm{u}$, with eigenvalues $\lambda_{k}(\mathrm{u}) \in \mathbb{R}, 1 \leq k \leq n$. For the numerical illustrations, we consider the $p$-system and the full compressible Euler system $(n=2$ or 3 in these cases). We propose an extension of the $D_{1} Q_{2}$ scheme compatible with the framework of the Multiple Relaxation Times lattice Boltzmann Schemes proposed by d'Humière [14].

\subsection{Description of the scheme}

We use the same notations for the regular lattice $\mathcal{L}$ with mesh size $\Delta x$. The time step $\Delta t$ is linked with the scheme velocity by the relation $\lambda=\Delta x / \Delta t$. Finally, the set of velocities $v$ is also defined by $v=(-\lambda, \lambda)$. The $\mathrm{D}_{1} \mathrm{Q}_{2}^{n}$ scheme is then defined by concatenate $n \mathrm{D}_{1} \mathrm{Q}_{2}$ schemes coupled through the equilibrium.

Let us introduce the particles distributions vector $f=\left(f_{1,0}, f_{1,1}, \ldots, f_{n, 0}, f_{n, 1}\right)^{\mathrm{T}}$ and the moments vector $\boldsymbol{m}=\left(\mathrm{u}_{1}, \ldots, \mathrm{u}_{n}, \mathrm{v}_{1}, \ldots, \mathrm{v}_{n}\right)^{\mathrm{T}}$. For the sake of readibility, we also define $\mathrm{u}=$ 
$\left(\mathrm{u}_{1}, \ldots, \mathrm{u}_{n}\right)^{\mathrm{T}}$ and $\mathrm{v}=\left(\mathrm{v}_{1}, \ldots, \mathrm{v}_{n}\right)^{\mathrm{T}}$. The matrix of the moments $\boldsymbol{M}$ then reads

$$
\boldsymbol{M}=\left(\begin{array}{ccccccc}
1 & 1 & 0 & 0 & \ldots & 0 & 0 \\
0 & 0 & \ddots & \ddots & & \vdots \\
\vdots & \ddots & \ddots & 0 & 0 \\
0 & 0 & \ldots & 0 & 0 & 1 & 1 \\
\lambda & \lambda & 0 & 0 & \ldots & 0 & 0 \\
0 & 0 & \ddots & \ddots & \vdots \\
\vdots & \ddots & \ddots & 0 & 0 \\
0 & 0 & \ldots & 0 & 0 & -\lambda & \lambda
\end{array}\right) .
$$

The inverse matrix $\boldsymbol{M}^{-1}$ is not given but can easily be obtained by the concatenatation of $n$ matrices corresponding to the scalar case. The starting point is the density vector $f(x, t)$ in $x \in \mathcal{L}$ at time $t$, the moments are then computed by

$$
\boldsymbol{m}(x, t)=\boldsymbol{M} \boldsymbol{f}(x, t) .
$$

The relaxation phase in the space of the moments reads

$$
\mathrm{u}_{k}^{\star}(x, t)=\mathrm{u}_{k}(x, t), \quad \mathrm{v}_{k}^{\star}(x, t)=\mathrm{v}_{k}(x, t)+s_{k}\left(\mathrm{v}_{k}^{\mathrm{eq}}(x, t)-\mathrm{v}_{k}(x, t)\right), \quad 1 \leq k \leq n,
$$

where $s_{k}, 1 \leq k \leq n$, is the $k$-th relaxation parameter and $\mathrm{v}_{k}^{\text {eq }}$ the moment at equilibrium that is a function of the vector $\mathrm{u}$. As a consequence, the first moment $\mathrm{u}$ is conserved during the relaxation phase. The densities are then computed by

$$
\boldsymbol{f}^{\star}(x, t)=\boldsymbol{M}^{-1} \boldsymbol{m}^{\star}(x, t) .
$$

The transport finally reads

$$
f_{k, j}(x, t+\Delta t)=f_{k, j}^{\star}\left(x-v_{j} \Delta t, t\right), \quad 0 \leq j \leq 1,1 \leq k \leq n .
$$

Concerning the treatment of the bounds, as the densities of each moment are decoupled, the standard Bouzidi conditions [18] can be applied independently on each moment: for instance, anti-bounce back conditions in order to impose first-order Dirichlet conditions. These simplicity is remarkable in particular for the full compressible Euler system for which the first and the third moments (corresponding to the mass and the energy) are usually coupled with standard lattice Boltzmann scheme like $D_{1} Q_{5}$ or more elaborated schemes with seven velocities for instance $[2,3]$.

\subsection{Asymptotic Analysis: the Taylor expansion method}

In this section, we use the Taylor expansion method to write the system of the equivalent equations as in section 1.2. No additional difficulties are involved by the dimension $n$.

Proposition 2.1 (zeroth order). Defining $\boldsymbol{m}^{\mathrm{eq}}=\left(\mathrm{u}_{1}, \ldots, \mathrm{u}_{n}, \mathrm{v}_{1}^{\mathrm{eq}}, \ldots, \mathrm{v}_{n}^{\mathrm{eq}}\right)$ and $\boldsymbol{f}^{\mathrm{eq}}=\boldsymbol{M}^{-1} \boldsymbol{m}^{\mathrm{eq}}$, we have

$$
f_{k, j}=f_{k, j}^{\mathrm{eq}}+O(\Delta t), \quad f_{k, j}^{\star}=f_{k, j}^{\mathrm{eq}}+O(\Delta t), \quad 0 \leq j \leq 1, \quad 1 \leq k \leq n .
$$


Proposition 2.2 (First order macroscopic equation). The first moments $\mathrm{u}=\left(\mathrm{u}_{1}, \ldots, \mathrm{u}_{n}\right)$ satisfies the partial differential equation

$$
\partial_{t} \mathrm{u}+\partial_{x} \mathrm{v}^{\mathrm{eq}}=O(\Delta t)
$$

with $\mathrm{v}^{\mathrm{eq}}=\left(\mathrm{v}_{1}^{\mathrm{eq}}, \ldots, \mathrm{v}_{n}^{\mathrm{eq}}\right)$. The choice $\mathrm{v}^{\mathrm{eq}}=\varphi(\mathrm{u})$ is then done so that $\mathrm{u}$ satisfies (19) at order 1 .

We then define the equilibrium default $\theta_{k}, 1 \leq k \leq n$, by using the particular derivatives $\mathrm{d}_{t}^{j}=\partial_{t}+v_{j} \partial_{x}, 0 \leq j \leq 1$,

$$
\theta_{k}=\sum_{j=0}^{1} v_{j} \mathrm{~d}_{t}^{j} f_{k, j}^{\mathrm{eq}}, \quad 1 \leq k \leq n .
$$

The equilibrium default $\theta_{k}$ can then be rewritten into the form

$$
\theta_{k}=\partial_{t} \mathrm{v}_{k}^{\mathrm{eq}}+\lambda^{2} \partial_{x} \mathrm{u}_{k}, \quad 1 \leq k \leq n .
$$

Lemma 2.3 (Transition lemma). The second moment $\mathrm{v}$ satisfies

$$
\mathrm{v}_{k}=\mathrm{v}_{k}^{\mathrm{eq}}-\frac{\Delta t}{s_{k}} \theta_{k}+O\left(\Delta t^{2}\right), \quad \mathrm{v}_{k}^{\star}=\mathrm{v}_{k}^{\mathrm{eq}}+\Delta t\left(1-\frac{1}{s_{k}}\right) \theta_{k}+O\left(\Delta t^{2}\right), \quad 1 \leq k \leq n .
$$

Moreover, we have

$$
f_{k, j}^{\star}-f_{k, j}=\Delta t \mathrm{~d}_{t}^{j} f_{k, j}^{\mathrm{eq}}+O\left(\Delta t^{2}\right), \quad 0 \leq j \leq 1, \quad 1 \leq k \leq n .
$$

Proposition 2.4 (Second order macroscopic equation). The first moment $\mathrm{u}$ satisfies the following system of second-order partial differential equations:

$$
\partial_{t} \mathrm{u}+\partial_{x} \varphi(\mathrm{u})=\Delta t \mathfrak{S} \partial_{x}\left(\left(\lambda^{2} \boldsymbol{I}_{n}-(\mathrm{d} \varphi(\mathrm{u}))^{2}\right) \partial_{x} \mathrm{u}\right)+O\left(\Delta t^{2}\right),
$$

with $\mathfrak{S}=\operatorname{diag}\left(\sigma_{1}, \ldots, \sigma_{n}\right), \sigma_{k}=1 / s_{k}-1 / 2,1 \leq k \leq n$, and $\boldsymbol{I}_{n}$ the identity matrix of size $n \times n$.

Let us remark that this system of second-order macroscopic equations (29) then contains a diffusion term with a regularization effect if $\sigma_{k}>0$ (that is $\left.s_{k}<2\right), 1 \leq k \leq n$, and $\left|\lambda_{k}(\mathrm{u})\right|<\lambda$, for $\lambda_{k}(\mathrm{u})$ eigenvalue of $\mathrm{d} \varphi(\mathrm{u})$.

\subsection{Link with the relaxation method}

Jin and Xin [7] extended the relaxation method to solve hyperbolic system of conservation laws by forming the linear system with a stiff source term :

$$
\left\{\begin{array}{c}
\partial_{t} \mathrm{u}^{\epsilon}+\partial_{x} \mathrm{v}^{\epsilon}=0, \\
\partial_{t} \mathrm{v}^{\epsilon}+A \partial_{x} \mathrm{u}^{\epsilon}=\frac{1}{\epsilon}\left(\varphi\left(\mathrm{u}^{\epsilon}\right)-\mathrm{v}^{\epsilon}\right),
\end{array}\right.
$$

where $A$ is a $n \times n$-dimensional matrix.

If all the relaxation parameters $s_{k}, 1 \leq k \leq n$, are equal to $s$ (BGK type lattice Boltzmann scheme), the $\mathrm{D}_{1} \mathrm{Q}_{2}^{n}$ scheme is then rewritten as a discretization of the relaxation system (30). Indeed, denoting $\mathrm{u}_{i}^{n}=\mathrm{u}\left(x_{i}, t^{n}\right), \mathrm{v}_{i}^{n}=\mathrm{v}\left(x_{i}, t^{n}\right), x_{i} \in \mathcal{L}$ and $t^{n}=n \Delta t$, relations $(15,16,17)$ are satisfied in a vectorial sens. We then reinterpret the scheme $\mathrm{D}_{1} \mathrm{Q}_{2}^{n}$ as a splitting between the relaxation part $(15)$ and the hyperbolic part $(16,17)$. The relaxation part is treated by the explicit Euler method with $\epsilon=\Delta t / s$, and the hyperbolic part by the Lax-Friedrichs method with $A=\lambda^{2} \boldsymbol{I}_{n}$. 
Moreover, as for the scalar case, the transport phase of the $D_{1} Q_{2}^{n}$ treats the hyperbolic part of the relaxation system (30) by an upwind scheme in the base of the eigenvectors.

The relaxation proposed by Jin and Xin does not imposed that $A$ is proportional to $\boldsymbol{I}_{n}$ (even if this particular case is specifically investigated). On the other hand, the stiff source term corresponding to the relaxation is proportional to $\boldsymbol{I}_{n}$ when the $\mathrm{D}_{1} \mathrm{Q}_{2}^{n}$ allows different values for the relaxation parameters.

\subsection{Numerical Illustrations}

In this section, we perform numerical illustrations for the $p$-system and the full Euler compressible equation. The lattice $\mathcal{L}$ is reduced to $[0,1]$ and homogeneous Neumann conditions are added to treat the boundaries. The initial condition is constant over $[0,0.5]$ and $] 0.5,1]$ in order to numerically solve the corresponding Riemann problem. We then denote $\mathrm{u}_{k L}$ and $\mathrm{u}_{k R}$ the left and the right value of the $k^{\text {th }}$ moment, so that we have at initial time

$$
\mathrm{u}_{k}(0, x)= \begin{cases}\mathrm{u}_{k L} & \text { if } x \leq 0.5 \\ \mathrm{u}_{k R} & \text { if } x>0.5 .\end{cases}
$$

The presented numerical results try to cover all the typical cases: the plots and the numerical convergence rates can be extended to all Riemann problems.

\subsection{1. p-system}

In this section, we consider the following $p$-system:

$$
\left\{\begin{array}{l}
\partial_{t} \mathrm{u}_{1}-\partial_{x} \mathrm{u}_{2}=0 \\
\partial_{t} \mathrm{u}_{2}-\partial_{x} p\left(\mathrm{u}_{1}\right)=0
\end{array}\right.
$$

where $p(\mathrm{u})=-\mathrm{u}^{-\gamma}$, with $\gamma=2 / 3$. This system of equations is hyperbolic as $\gamma>0$ and the eigenvalues of the jacobian matrix are $\pm \sqrt{\gamma} \mathrm{u}^{-\frac{\gamma+1}{2}}$.

In Fig. 3 (resp. Fig. 4), the two figures show the initial and the final (at time $T=0.3$ ) moments $\mathrm{u}_{1}$ and $\mathrm{u}_{2}$ for several relaxation parameters $s_{1}, s_{2}$, where the initial conditions are chosen in order to obtain 1-shock, 2-rarefaction waves (resp. 1-rarefaction, 2-shock waves). For the numerical values, we have the velocity of the scheme $\lambda=1$, the number of points $N=200$, and the initial condition given by

- for the 1-shock, 2-rarefaction: $\mathrm{u}_{1 L}=1.5, \mathrm{u}_{2 L}=1.25, \mathrm{u}_{1 R}=1.0, \mathrm{u}_{2 R}=1.0$,

- for the 1-rarefaction, 2-shock: $\mathrm{u}_{1 L}=1.0, \mathrm{u}_{2 L}=1.0, \mathrm{u}_{1 R}=1.5, \mathrm{u}_{2 R}=1.25$.

The Tbl. 5 (resp. Tbl. 6) shows the convergence of the $L^{2}$-norm for several relaxation parameters $s_{1}, s_{2}$ when $\Delta x$ goes to zero for the 1-shock, 2-rarefaction waves (resp. 1-rarefaction, 2-shock waves). Each line corresponds to the integer $k \in\{3, \ldots, 16\}$ with $\Delta x=2^{-k}$. Essentially, we observe a convergence at order 0.5 due to the discontinuity of the solution. In the case of 1rarefaction, 2-rarefaction waves (the solution is then continuous for $t>0$ ), the same investigation yields to a higher order, between 0.64 and 0.8 depending on the relaxation parameters. 

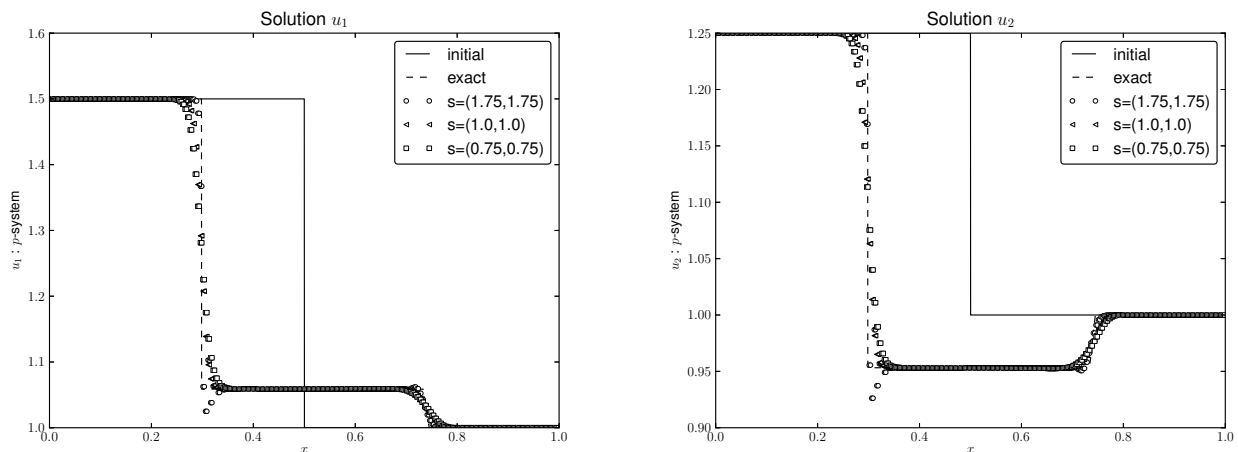

Figure 3: $p$-system at final time $T=0.3$ (left: $\mathrm{u}_{1}$, right: $\mathrm{u}_{2}$ ) Riemann problem corresponding to 1-shock, 2-rarefaction
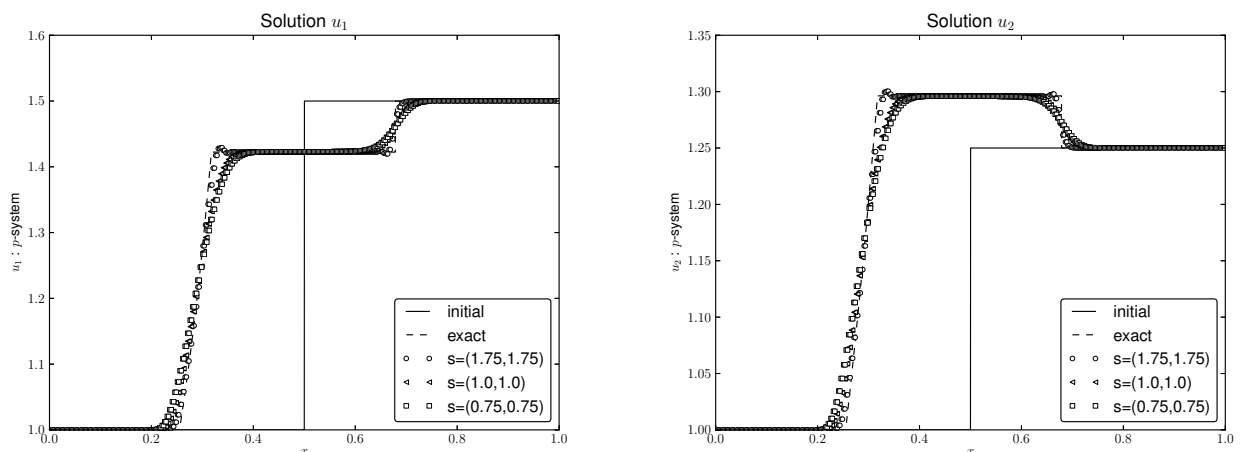

Figure 4: $p$-system at final time $T=0.3$ (left: $\mathrm{u}_{1}$, right: $\mathrm{u}_{2}$ ) Riemann problem corresponding to 1-rarefaction, 2-shock 


\begin{tabular}{|c|c|c|c|c|c|c|c|}
\hline $\begin{array}{r}s_{1} \\
k s_{2}\end{array}$ & $\begin{array}{l}0.500 \\
0.500\end{array}$ & $\begin{array}{l}1.000 \\
1.000\end{array}$ & $\begin{array}{l}1.500 \\
1.500\end{array}$ & $\begin{array}{l}1.900 \\
1.900\end{array}$ & $\begin{array}{l}0.500 \\
1.000\end{array}$ & $\begin{array}{l}1.000 \\
0.500\end{array}$ & $\begin{array}{l}1.500 \\
1.000\end{array}$ \\
\hline 3 & $1.527 \mathrm{e}-01$ & $1.428 \mathrm{e}-01$ & $1.379 \mathrm{e}-01$ & $1.378 \mathrm{e}-01$ & $1.497 \mathrm{e}-01$ & $1.459 \mathrm{e}-01$ & $1.396 \mathrm{e}-01$ \\
\hline 4 & $1.057 \mathrm{e}-01$ & $9.754 \mathrm{e}-02$ & $1.007 \mathrm{e}-01$ & $1.154 \mathrm{e}-01$ & $1.030 \mathrm{e}-01$ & $1.012 \mathrm{e}-01$ & $9.777 \mathrm{e}-02$ \\
\hline 5 & $9.398 \mathrm{e}-02$ & $7.297 \mathrm{e}-02$ & $6.757 \mathrm{e}-02$ & $7.972 \mathrm{e}-02$ & $8.562 \mathrm{e}-02$ & $8.371 \mathrm{e}-02$ & $6.930 \mathrm{e}-02$ \\
\hline 6 & $7.163 \mathrm{e}-02$ & $4.956 \mathrm{e}-02$ & $3.718 \mathrm{e}-02$ & $5.121 \mathrm{e}-02$ & $6.313 \mathrm{e}-02$ & $6.259 \mathrm{e}-02$ & $4.366 \mathrm{e}-02$ \\
\hline 7 & $5.801 \mathrm{e}-02$ & $4.057 \mathrm{e}-02$ & $3.138 \mathrm{e}-02$ & $4.426 \mathrm{e}-02$ & $5.111 \mathrm{e}-02$ & $5.102 \mathrm{e}-02$ & $3.605 \mathrm{e}-02$ \\
\hline 8 & $4.754 \mathrm{e}-02$ & $3.313 \mathrm{e}-02$ & $2.520 \mathrm{e}-02$ & $3.552 \mathrm{e}-02$ & $4.177 \mathrm{e}-02$ & $4.158 \mathrm{e}-02$ & $2.925 \mathrm{e}-02$ \\
\hline 9 & $3.697 \mathrm{e}-02$ & $2.376 \mathrm{e}-02$ & $1.496 \mathrm{e}-02$ & $1.721 \mathrm{e}-02$ & $3.177 \mathrm{e}-02$ & $3.171 \mathrm{e}-02$ & $1.986 \mathrm{e}-02$ \\
\hline 10 & $2.859 \mathrm{e}-02$ & $1.803 \mathrm{e}-02$ & $1.274 \mathrm{e}-02$ & $1.776 \mathrm{e}-02$ & $2.425 \mathrm{e}-02$ & $2.418 \mathrm{e}-02$ & $1.534 \mathrm{e}-02$ \\
\hline 11 & $2.118 \mathrm{e}-02$ & $1.287 \mathrm{e}-02$ & $9.210 \mathrm{e}-03$ & $1.192 \mathrm{e}-02$ & $1.764 \mathrm{e}-02$ & $1.763 \mathrm{e}-02$ & $1.098 \mathrm{e}-02$ \\
\hline 12 & $1.520 \mathrm{e}-02$ & $8.813 \mathrm{e}-03$ & $5.519 \mathrm{e}-03$ & $6.085 \mathrm{e}-03$ & $1.246 \mathrm{e}-02$ & $1.246 \mathrm{e}-02$ & $7.262 \mathrm{e}-03$ \\
\hline 13 & $1.083 \mathrm{e}-02$ & $6.426 \mathrm{e}-03$ & $4.486 \mathrm{e}-03$ & $6.092 \mathrm{e}-03$ & $8.899 \mathrm{e}-03$ & $8.889 \mathrm{e}-03$ & $5.436 \mathrm{e}-03$ \\
\hline 14 & $7.638 \mathrm{e}-03$ & $4.376 \mathrm{e}-03$ & $2.539 \mathrm{e}-03$ & $2.883 \mathrm{e}-03$ & $6.237 \mathrm{e}-03$ & $6.233 \mathrm{e}-03$ & $3.553 \mathrm{e}-03$ \\
\hline 15 & $5.402 \mathrm{e}-03$ & $3.088 \mathrm{e}-03$ & $1.769 \mathrm{e}-03$ & $2.052 \mathrm{e}-03$ & $4.408 \mathrm{e}-03$ & $4.404 \mathrm{e}-03$ & $2.502 \mathrm{e}-03$ \\
\hline 16 & $3.816 \mathrm{e}-03$ & $2.178 \mathrm{e}-03$ & $1.226 \mathrm{e}-03$ & $1.500 \mathrm{e}-03$ & $3.112 \mathrm{e}-03$ & $3.108 \mathrm{e}-03$ & $1.760 \mathrm{e}-03$ \\
\hline slope & $5.014 \mathrm{e}-01$ & $3.0530-01$ & $3.2000-01$ & $4.519 \mathrm{e}-01$ & $5.021 \mathrm{e}-01$ & $5.027 \mathrm{e}-01$ & $5.073 \mathrm{e}-01$ \\
\hline$s_{1}$ & 1.000 & 1.9 & 1.000 & & 1.500 & & 1.900 \\
\hline$k s_{2}$ & 1.500 & 1.000 & 1.900 & 0 & 0.500 & 1.900 & 1.500 \\
\hline 3 & $1.412 \mathrm{e}-01$ & $1.397 \mathrm{e}-01$ & $1.409 \mathrm{e}-01$ & $1.481 \mathrm{e}-01$ & $1.427 \mathrm{e}-01$ & $1.376 \mathrm{e}-01$ & $1.380 \mathrm{e}-01$ \\
\hline 4 & $9.755 \mathrm{e}-02$ & $9.936 \mathrm{e}-02$ & $9.863 \mathrm{e}-02$ & $1.025 \mathrm{e}-01$ & $1.014 \mathrm{e}-01$ & $1.052 \mathrm{e}-01$ & $1.056 \mathrm{e}-01$ \\
\hline 5 & $6.961 \mathrm{e}-02$ & $6.835 \mathrm{e}-02$ & $6.861 \mathrm{e}-02$ & $8.253 \mathrm{e}-02$ & $8.053 \mathrm{e}-02$ & $6.968 \mathrm{e}-02$ & $6.974 \mathrm{e}-02$ \\
\hline 6 & $4.346 \mathrm{e}-02$ & $4.156 \mathrm{e}-02$ & $4.100 \mathrm{e}-02$ & $5.978 \mathrm{e}-02$ & $5.937 \mathrm{e}-02$ & $3.790 \mathrm{e}-02$ & $3.841 \mathrm{e}-02$ \\
\hline 7 & $3.581 \mathrm{e}-02$ & $3.443 \mathrm{e}-02$ & $3.392 \mathrm{e}-02$ & $4.836 \mathrm{e}-02$ & $4.841 \mathrm{e}-02$ & $3.210 \mathrm{e}-02$ & $3.245 \mathrm{e}-02$ \\
\hline 8 & $2.928 \mathrm{e}-02$ & $2.756 \mathrm{e}-02$ & $2.757 \mathrm{e}-02$ & $3.939 \mathrm{e}-02$ & $3.918 \mathrm{e}-02$ & $2.468 \mathrm{e}-02$ & $2.471 \mathrm{e}-02$ \\
\hline 9 & $1.982 \mathrm{e}-02$ & $1.805 \mathrm{e}-02$ & $1.795 \mathrm{e}-02$ & $2.960 \mathrm{e}-02$ & $2.956 \mathrm{e}-02$ & $1.372 \mathrm{e}-02$ & $1.385 \mathrm{e}-02$ \\
\hline 10 & $1.539 \mathrm{e}-02$ & $1.417 \mathrm{e}-02$ & $1.424 \mathrm{e}-02$ & $2.248 \mathrm{e}-02$ & $2.239 \mathrm{e}-02$ & $1.244 \mathrm{e}-02$ & $1.241 \mathrm{e}-02$ \\
\hline 11 & $1.096 \mathrm{e}-02$ & $1.023 \mathrm{e}-02$ & $1.019 \mathrm{e}-02$ & $1.625 \mathrm{e}-02$ & $1.624 \mathrm{e}-02$ & $8.956 \mathrm{e}-03$ & $8.980 \mathrm{e}-03$ \\
\hline 12 & $7.241 \mathrm{e}-03$ & $6.595 e-03$ & $6.543 \mathrm{e}-03$ & $1.141 \mathrm{e}-02$ & $1.142 \mathrm{e}-02$ & $5.173 \mathrm{e}-03$ & $5.222 \mathrm{e}-03$ \\
\hline 13 & $5.441 \mathrm{e}-03$ & $5.023 \mathrm{e}-03$ & $5.027 \mathrm{e}-03$ & $8.164 \mathrm{e}-03$ & $8.153 \mathrm{e}-03$ & $4.348 \mathrm{e}-03$ & $4.346 \mathrm{e}-03$ \\
\hline 14 & $3.552 \mathrm{e}-03$ & $3.173 \mathrm{e}-03$ & $3.163 \mathrm{e}-03$ & $5.698 \mathrm{e}-03$ & $5.695 \mathrm{e}-03$ & $2.207 \mathrm{e}-03$ & $2.224 \mathrm{e}-03$ \\
\hline 15 & $2.502 \mathrm{e}-03$ & $2.227 \mathrm{e}-03$ & $2.223 \mathrm{e}-03$ & $4.025 \mathrm{e}-03$ & $4.022 \mathrm{e}-03$ & $1.511 \mathrm{e}-03$ & $1.521 \mathrm{e}-03$ \\
\hline 16 & $1.762 \mathrm{e}-03$ & $1.560 \mathrm{e}-03$ & $1.561 \mathrm{e}-03$ & $2.841 \mathrm{e}-03$ & $2.836 \mathrm{e}-03$ & $1.014 \mathrm{e}-03$ & $1.017 \mathrm{e}-03$ \\
\hline slope & $5.055 \mathrm{e}-01$ & $5.137 \mathrm{e}-01$ & $5.098 \mathrm{e}-01$ & $5.027 \mathrm{e}-01$ & $5.037 \mathrm{e}-01$ & $5.757 \mathrm{e}-01$ & $5.798 \mathrm{e}-01$ \\
\hline
\end{tabular}

Table 5: $p$-system at final time $T=0.3$ (1-shock, 2-rarefaction: error in $L^{2}$ norm) 


\begin{tabular}{|c|c|c|c|c|c|c|c|}
\hline $\begin{array}{r}s_{1} \\
k \quad s_{2}\end{array}$ & $\begin{array}{l}0.500 \\
0.500\end{array}$ & $\begin{array}{l}1.000 \\
1.000\end{array}$ & $\begin{array}{l}1.500 \\
1.500\end{array}$ & $\begin{array}{l}1.900 \\
1.900\end{array}$ & $\begin{array}{l}0.500 \\
1.000\end{array}$ & $\begin{array}{l}1.000 \\
0.500\end{array}$ & $\begin{array}{l}1.500 \\
1.000\end{array}$ \\
\hline 3 & $1.105 \mathrm{e}-01$ & $1.016 \mathrm{e}-01$ & $9.836 \mathrm{e}-02$ & $1.003 \mathrm{e}-01$ & $1.092 \mathrm{e}-01$ & $1.030 \mathrm{e}-01$ & $9.895 \mathrm{e}-02$ \\
\hline 4 & $1.005 \mathrm{e}-01$ & $8.795 \mathrm{e}-02$ & $8.294 \mathrm{e}-02$ & $8.454 \mathrm{e}-02$ & $9.734 \mathrm{e}-02$ & $9.316 \mathrm{e}-02$ & $8.549 \mathrm{e}-02$ \\
\hline 5 & $8.301 \mathrm{e}-02$ & $6.054 \mathrm{e}-02$ & $4.975 \mathrm{e}-02$ & $5.020 \mathrm{e}-02$ & $7.503 \mathrm{e}-02$ & $7.365 \mathrm{e}-02$ & $5.544 \mathrm{e}-02$ \\
\hline 6 & $6.483 \mathrm{e}-02$ & $4.451 \mathrm{e}-02$ & $3.236 \mathrm{e}-02$ & $2.993 \mathrm{e}-02$ & $5.678 \mathrm{e}-02$ & $5.661 \mathrm{e}-02$ & $3.924 \mathrm{e}-02$ \\
\hline 7 & $4.948 \mathrm{e}-02$ & $3.265 \mathrm{e}-02$ & $2.172 \mathrm{e}-02$ & $1.831 \mathrm{e}-02$ & $4.262 \mathrm{e}-02$ & $4.283 \mathrm{e}-02$ & $2.807 \mathrm{e}-02$ \\
\hline 8 & $3.807 \mathrm{e}-02$ & $2.425 \mathrm{e}-02$ & $1.513 \mathrm{e}-02$ & $1.113 \mathrm{e}-02$ & $3.244 \mathrm{e}-02$ & $3.253 \mathrm{e}-02$ & $2.040 \mathrm{e}-02$ \\
\hline 9 & $2.870 \mathrm{e}-02$ & $1.753 \mathrm{e}-02$ & $1.034 \mathrm{e}-02$ & $6.624 \mathrm{e}-03$ & $2.408 \mathrm{e}-02$ & $2.416 \mathrm{e}-02$ & $1.448 \mathrm{e}-02$ \\
\hline 10 & $2.114 \mathrm{e}-02$ & $1.243 \mathrm{e}-02$ & $7.105 \mathrm{e}-03$ & $4.193 \mathrm{e}-03$ & $1.747 \mathrm{e}-02$ & $1.753 \mathrm{e}-02$ & $1.015 \mathrm{e}-02$ \\
\hline 11 & $1.519 \mathrm{e}-02$ & $8.681 \mathrm{e}-03$ & $4.883 \mathrm{e}-03$ & $2.745 \mathrm{e}-03$ & $1.240 \mathrm{e}-02$ & $1.244 \mathrm{e}-02$ & $7.032 \mathrm{e}-03$ \\
\hline 12 & $1.071 \mathrm{e}-02$ & $6.009 \mathrm{e}-03$ & $3.363 \mathrm{e}-03$ & $1.755 \mathrm{e}-03$ & $8.662 \mathrm{e}-03$ & $8.684 \mathrm{e}-03$ & $4.852 \mathrm{e}-03$ \\
\hline 13 & $7.445 \mathrm{e}-03$ & $4.150 \mathrm{e}-03$ & $2.312 \mathrm{e}-03$ & $1.158 \mathrm{e}-03$ & $5.997 \mathrm{e}-03$ & $6.011 \mathrm{e}-03$ & $3.349 \mathrm{e}-03$ \\
\hline 14 & $5.149 \mathrm{e}-03$ & $2.869 \mathrm{e}-03$ & $1.586 \mathrm{e}-03$ & $7.763 \mathrm{e}-04$ & $4.146 \mathrm{e}-03$ & $4.153 \mathrm{e}-03$ & $2.312 \mathrm{e}-03$ \\
\hline 15 & $3.560 \mathrm{e}-03$ & $1.978 \mathrm{e}-03$ & $1.081 \mathrm{e}-03$ & $5.688 \mathrm{e}-04$ & $2.867 \mathrm{e}-03$ & $2.872 \mathrm{e}-03$ & $1.587 \mathrm{e}-03$ \\
\hline 16 & $2.460 \mathrm{e}-03$ & $1.350 \mathrm{e}-03$ & $7.328 \mathrm{e}-04$ & $3.875 \mathrm{e}-04$ & $1.976 \mathrm{e}-03$ & $1.979 \mathrm{e}-03$ & $1.078 \mathrm{e}-03$ \\
\hline slope & $5.332 \mathrm{e}-01$ & $5.505 \mathrm{e}-01$ & $5.607 \mathrm{e}-01$ & $5.540 \mathrm{e}-01$ & $5.373 \mathrm{e}-01$ & $5.373 \mathrm{e}-01$ & $5.575 \mathrm{e}-01$ \\
\hline$s_{1}$ & 1.000 & 1.900 & 1.000 & 0.500 & 1.500 & 1.500 & 1.900 \\
\hline$k s_{2}$ & 1.500 & 1.000 & 1.900 & 1. & 00 & 00 & 1.500 \\
\hline 3 & $1.010 \mathrm{e}-01$ & $1.007 \mathrm{e}-01$ & $1.011 \mathrm{e}-01$ & $1.087 \mathrm{e}-01$ & $1.004 \mathrm{e}-01$ & $9.851 \mathrm{e}-02$ & $1.002 \mathrm{e}-01$ \\
\hline 4 & $8.547 \mathrm{e}-02$ & $8.633 \mathrm{e}-02$ & $8.467 \mathrm{e}-02$ & $9.583 \mathrm{e}-02$ & $9.263 \mathrm{e}-02$ & $8.289 \mathrm{e}-02$ & $8.331 \mathrm{e}-02$ \\
\hline 5 & $5.515 \mathrm{e}-02$ & $5.375 \mathrm{e}-02$ & $5.306 \mathrm{e}-02$ & $7.179 \mathrm{e}-02$ & $7.116 \mathrm{e}-02$ & $4.854 \mathrm{e}-02$ & $4.900 \mathrm{e}-02$ \\
\hline 6 & $3.906 \mathrm{e}-02$ & $3.697 \mathrm{e}-02$ & $3.654 \mathrm{e}-02$ & $5.364 \mathrm{e}-02$ & $5.374 \mathrm{e}-02$ & $2.979 \mathrm{e}-02$ & $3.013 \mathrm{e}-02$ \\
\hline 7 & $2.783 \mathrm{e}-02$ & $2.593 \mathrm{e}-02$ & $2.547 \mathrm{e}-02$ & $3.988 \mathrm{e}-02$ & $4.031 \mathrm{e}-02$ & $1.885 \mathrm{e}-02$ & $1.916 \mathrm{e}-02$ \\
\hline 8 & $2.032 \mathrm{e}-02$ & $1.851 \mathrm{e}-02$ & $1.836 \mathrm{e}-02$ & $3.016 \mathrm{e}-02$ & $3.035 \mathrm{e}-02$ & $1.249 \mathrm{e}-02$ & $1.259 \mathrm{e}-02$ \\
\hline 9 & $1.443 \mathrm{e}-02$ & $1.297 \mathrm{e}-02$ & $1.287 \mathrm{e}-02$ & $2.222 \mathrm{e}-02$ & $2.235 \mathrm{e}-02$ & $8.136 \mathrm{e}-03$ & $8.207 \mathrm{e}-03$ \\
\hline 10 & $1.011 \mathrm{e}-02$ & $9.021 \mathrm{e}-03$ & $8.952 \mathrm{e}-03$ & $1.600 \mathrm{e}-02$ & $1.610 \mathrm{e}-02$ & $5.461 \mathrm{e}-03$ & $5.512 \mathrm{e}-03$ \\
\hline 11 & $7.007 \mathrm{e}-03$ & $6.227 \mathrm{e}-03$ & $6.183 e-03$ & $1.130 \mathrm{e}-02$ & $1.136 \mathrm{e}-02$ & $3.712 \mathrm{e}-03$ & $3.746 \mathrm{e}-03$ \\
\hline 12 & $4.842 \mathrm{e}-03$ & $4.287 \mathrm{e}-03$ & $4.271 \mathrm{e}-03$ & $7.869 \mathrm{e}-03$ & $7.900 \mathrm{e}-03$ & $2.540 \mathrm{e}-03$ & $2.553 \mathrm{e}-03$ \\
\hline 13 & $3.342 \mathrm{e}-03$ & $2.958 \mathrm{e}-03$ & $2.945 \mathrm{e}-03$ & $5.441 \mathrm{e}-03$ & $5.461 \mathrm{e}-03$ & $1.727 \mathrm{e}-03$ & $1.738 \mathrm{e}-03$ \\
\hline 14 & $2.308 \mathrm{e}-03$ & $2.037 \mathrm{e}-03$ & $2.032 \mathrm{e}-03$ & $3.762 \mathrm{e}-03$ & $3.772 \mathrm{e}-03$ & $1.178 \mathrm{e}-03$ & $1.182 \mathrm{e}-03$ \\
\hline 15 & $1.583 \mathrm{e}-03$ & $1.395 \mathrm{e}-03$ & $1.389 \mathrm{e}-03$ & $2.600 \mathrm{e}-03$ & $2.608 \mathrm{e}-03$ & $8.064 \mathrm{e}-04$ & $8.126 \mathrm{e}-04$ \\
\hline 16 & $1.075 \mathrm{e}-03$ & $9.464 \mathrm{e}-04$ & $9.415 \mathrm{e}-04$ & $1.788 \mathrm{e}-03$ & $1.793 \mathrm{e}-03$ & $5.473 \mathrm{e}-04$ & $5.525 \mathrm{e}-04$ \\
\hline slope & $5.581 \mathrm{e}-01$ & $5.595 \mathrm{e}-01$ & $5.609 \mathrm{e}-01$ & $5.403 \mathrm{e}-01$ & $5.401 \mathrm{e}-01$ & $5.591 \mathrm{e}-01$ & $5.565 \mathrm{e}-01$ \\
\hline
\end{tabular}

Table 6: $p$-system at final time $T=0.3$ (1-rarefaction, 2-shock: error in $L^{2}$ norm) 


\subsubsection{Full compressible Euler system}

In this section, the $\mathrm{D}_{1} \mathrm{Q}_{2}^{n}$ scheme is tested to simulate the mono-dimensional Euler equations

$$
\left\{\begin{array}{l}
\partial_{t} \rho+\partial_{x}(\rho u)=0, \\
\partial_{t}(\rho u)+\partial_{x}\left(\rho u^{2}+p\right)=0, \\
\partial_{t} E+\partial_{x}(E u+p u)=0,
\end{array}\right.
$$

where $\rho$ is the mass, $u$ the velocity, $E=\rho u^{2}+p /(\gamma-1)$ the energy, and $p$ the pressure. The Euler equations can then be viewed as a conservative hyperbolic system in the variable $\mathrm{u}_{1}=\rho$, $\mathrm{u}_{2}=\rho u$, and $\mathrm{u}_{3}=E$. For the numerical simulations, the test case is the Sod shock tube $\left(\rho_{L}=1.0\right.$, $\left.p_{L}=1.0, u_{L}=0.0, \rho_{R}=0.125, p_{R}=0.1, u_{R}=0.0\right), \gamma$ is taken to 1.4 , the number of points $N=800$, and the scheme velocity is $\lambda=3.0$.

In Fig. 5, the mass $\rho$ is plotted at time $T=0.2$ for several relaxation parameters: $s_{1}=s_{2}=$ $s_{3}=s$, with $s \in\{0.5,0.75,1.0,1.5,1.75,1.9\}$. The numerical diffusion is as expected higher for small relaxation parameters, whereas numerical oscillations are observed for large relaxation parameters (after the shock wave and also after the contact discontinuity).
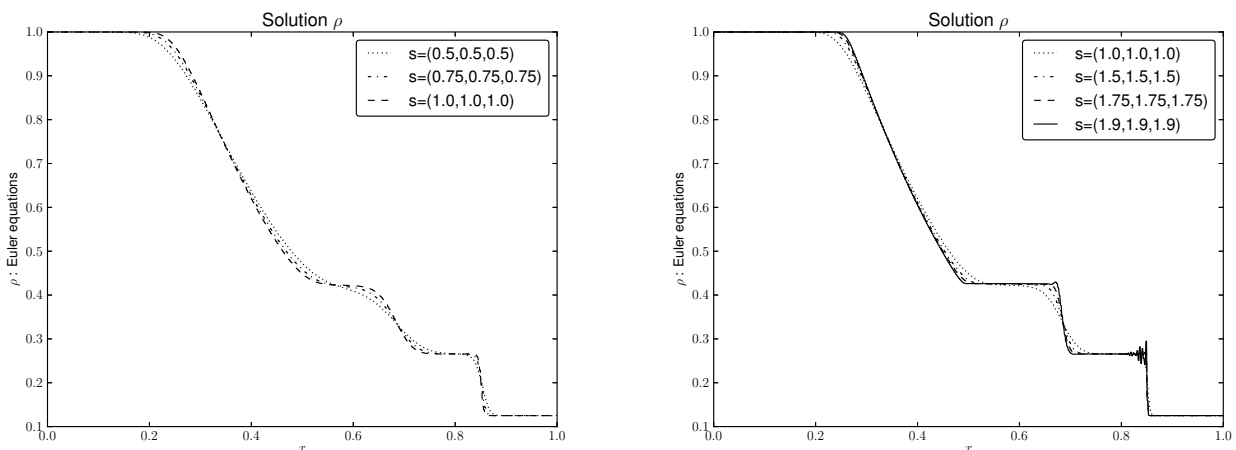

Figure 5: Euler system at final time $T=0.2$ (left: $s \leq 1$, right: $s \geq 1$ ) Sod Shock Tube

Numerical convergence results in $L^{2}$ norm are given in Tbl. 7 for several relaxation parameters $s_{1}, s_{2}$, and $s_{3}$ when $\Delta x$ goes to zero, each line corresponding to the integer $k \in\{3, \ldots, 16\}$ with $\Delta x=2^{-k}$. The error in $L^{2}$ norm goes to zero with an order that depends on the relaxation parameters. The convergence seems to be quicker when the three relaxation parameters move nearer to 2, the order approaching 0.5 .

In Fig. 6, the mass, the velocity, and the pressure are plotted, the exact solution with a solid line and the approximate one with a dashed line. The parameters of this simulation are $N=1000$, $T=0.14, s_{1}=1.9, s_{2}=1.5$, and $s_{3}=1.4$. It appears as a good compromise between numerical diffusion and oscillations in the area of discontinuities.

\section{Appendix A. Taylor expansion method for the scalar case}

The Taylor expansion method consists in expanding the distribution functions with respect to the small parameter $\Delta t$. Considering Eq. (8), we have

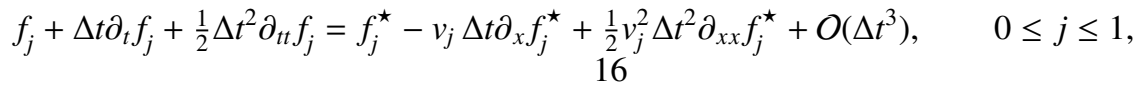




\begin{tabular}{lcccccc}
\hline$s_{1}$ & 1.900 & 0.500 & 1.000 & 1.500 & 1.900 & 1.990 \\
$s_{2}$ & 1.500 & 0.500 & 1.000 & 1.500 & 1.900 & 1.990 \\
$k s_{3}$ & 1.400 & 0.500 & 1.000 & 1.500 & 1.900 & 1.990 \\
\hline 3 & $1.297 \mathrm{e}-01$ & $1.709 \mathrm{e}-01$ & $1.278 \mathrm{e}-01$ & $1.133 \mathrm{e}-01$ & $1.297 \mathrm{e}-01$ & $1.361 \mathrm{e}-01$ \\
4 & $9.789 \mathrm{e}-02$ & $1.242 \mathrm{e}-01$ & $8.141 \mathrm{e}-02$ & $7.408 \mathrm{e}-02$ & $8.542 \mathrm{e}-02$ & $9.103 \mathrm{e}-02$ \\
5 & $6.229 \mathrm{e}-02$ & $8.670 \mathrm{e}-02$ & $5.454 \mathrm{e}-02$ & $4.380 \mathrm{e}-02$ & $4.041 \mathrm{e}-02$ & $4.230 \mathrm{e}-02$ \\
6 & $4.795 \mathrm{e}-02$ & $6.764 \mathrm{e}-02$ & $4.643 \mathrm{e}-02$ & $3.445 \mathrm{e}-02$ & $2.644 \mathrm{e}-02$ & $3.112 \mathrm{e}-02$ \\
7 & $3.136 \mathrm{e}-02$ & $5.136 \mathrm{e}-02$ & $3.691 \mathrm{e}-02$ & $2.528 \mathrm{e}-02$ & $1.639 \mathrm{e}-02$ & $2.358 \mathrm{e}-02$ \\
8 & $2.205 \mathrm{e}-02$ & $4.132 \mathrm{e}-02$ & $2.951 \mathrm{e}-02$ & $1.957 \mathrm{e}-02$ & $1.377 \mathrm{e}-02$ & $2.085 \mathrm{e}-02$ \\
9 & $1.421 \mathrm{e}-02$ & $3.369 \mathrm{e}-02$ & $2.229 \mathrm{e}-02$ & $1.416 \mathrm{e}-02$ & $9.334 \mathrm{e}-03$ & $1.944 \mathrm{e}-02$ \\
10 & $1.008 \mathrm{e}-02$ & $2.645 \mathrm{e}-02$ & $1.651 \mathrm{e}-02$ & $1.023 \mathrm{e}-02$ & $6.232 \mathrm{e}-03$ & $1.591 \mathrm{e}-02$ \\
11 & $7.191 \mathrm{e}-03$ & $1.974 \mathrm{e}-02$ & $1.220 \mathrm{e}-02$ & $7.995 \mathrm{e}-03$ & $5.324 \mathrm{e}-03$ & $1.173 \mathrm{e}-02$ \\
12 & $5.129 \mathrm{e}-03$ & $1.452 \mathrm{e}-02$ & $9.122 \mathrm{e}-03$ & $6.169 \mathrm{e}-03$ & $4.011 \mathrm{e}-03$ & $8.173 \mathrm{e}-03$ \\
13 & $3.903 \mathrm{e}-03$ & $1.080 \mathrm{e}-02$ & $7.035 \mathrm{e}-03$ & $4.876 \mathrm{e}-03$ & $3.069 \mathrm{e}-03$ & $6.121 \mathrm{e}-03$ \\
14 & $3.011 \mathrm{e}-03$ & $8.179 \mathrm{e}-03$ & $5.547 \mathrm{e}-03$ & $3.980 \mathrm{e}-03$ & $2.531 \mathrm{e}-03$ & $4.304 \mathrm{e}-03$ \\
15 & $2.443 \mathrm{e}-03$ & $6.363 \mathrm{e}-03$ & $4.484 \mathrm{e}-03$ & $3.301 \mathrm{e}-03$ & $2.163 \mathrm{e}-03$ & $3.177 \mathrm{e}-03$ \\
16 & $1.968 \mathrm{e}-03$ & $5.064 \mathrm{e}-03$ & $3.665 \mathrm{e}-03$ & $2.738 \mathrm{e}-03$ & $1.769 \mathrm{e}-03$ & $2.259 \mathrm{e}-03$ \\
\hline slope & $3.119 \mathrm{e}-01$ & $3.296 \mathrm{e}-01$ & $2.908 \mathrm{e}-01$ & $2.699 \mathrm{e}-01$ & $2.901 \mathrm{e}-01$ & $4.924 \mathrm{e}-01$ \\
\hline
\end{tabular}

Table 7: Sod shock tube at final time $T=0.1$ (error in $L^{2}$ norm)
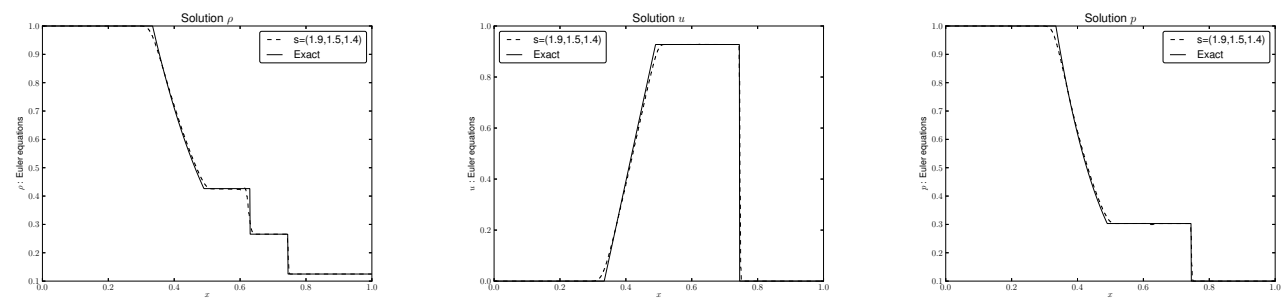

Figure 6: Solution du systme d'Euler l'instant final $t=0.14$ pour un tube choc de Sod 
where the variables $x \in \mathcal{L}$ and $t$ have been removed for readability. As the relaxation phase is written in the space of moments, we immediately take the moments of order 0 and $1 \mathrm{of} \mathrm{Eq.} \mathrm{(A.1)}$ by summing over $j$ after multiplication by $v_{j}^{0}$ or $v_{j}^{1}$ :

$$
\begin{array}{cc}
\mathrm{u}+\Delta t \partial_{t} \mathrm{u}+\frac{1}{2} \Delta t^{2} \partial_{t t \mathrm{u}} \mathrm{u}=\mathrm{u}^{\star}-\Delta t \partial_{x} \mathrm{v}^{\star}+\frac{\lambda^{2}}{2} \Delta t^{2} \partial_{x x} \mathrm{u}^{\star}+O\left(\Delta t^{3}\right), & 0 \leq j \leq 1, \\
\mathrm{v}+\Delta t \partial_{t} \mathrm{v}+\frac{1}{2} \Delta t^{2} \partial_{t t} \mathrm{v}=\mathrm{v}^{\star}-\lambda^{2} \Delta t \partial_{x} \mathrm{u}^{\star}+\frac{\lambda^{2}}{2} \Delta t^{2} \partial_{x x} \mathrm{v}^{\star}+O\left(\Delta t^{3}\right), & 0 \leq j \leq 1 .
\end{array}
$$

We then consider Eqs. (A.2) and (A.3) at order $k$ for $0 \leq k \leq 2$.

- Eq. (A.2) at zeroth-order does not give information: as the first moment $u$ is conserved during the relaxation phase, $\mathrm{u}=\mathrm{u}^{\star}$.

- Eq. (A.3) at zeroth-order reads $\mathrm{v}=\mathrm{v}^{\star}+O(\Delta t)$. Using Eq. (6) $\mathrm{v}^{\star}=\mathrm{v}+s\left(\mathrm{v}^{\mathrm{eq}}-\mathrm{v}\right)$, it yields to Eq. (9)

$$
\mathrm{v}=\mathrm{v}^{\mathrm{eq}}+O(\Delta t), \quad \mathrm{v}^{\star}=\mathrm{v}^{\mathrm{eq}}+O(\Delta t),
$$

as the relaxation parameter $s$ is considered as a constant.

- Eq. (A.2) at first-order (after division by $\Delta t$ ) can be rewritten in the form

$$
\partial_{t} \mathrm{u}+\partial_{x} \mathrm{v}^{\mathrm{eq}}=O(\Delta t)
$$

by using (9).

- Eq. (A.3) at first-order reads

$$
\mathrm{v}^{\star}-\mathrm{v}=\Delta t\left(\partial_{t} \mathrm{v}^{\mathrm{eq}}+\lambda^{2} \partial_{x} \mathrm{u}\right)+O\left(\Delta t^{2}\right)=\Delta t \theta+O\left(\Delta t^{2}\right),
$$

by using the definition of the equilibrium default (11). Combining this equation with Eq. (6) then yields

$$
\mathrm{v}=\mathrm{v}^{\mathrm{eq}}-\frac{\Delta t}{s} \theta+O\left(\Delta t^{2}\right), \quad \mathrm{v}^{\star}=\mathrm{v}^{\mathrm{eq}}+\Delta t\left(1-\frac{1}{s}\right) \theta+O\left(\Delta t^{2}\right) .
$$

- Eq. (A.2) at second-order reads

$$
\partial_{t} \mathrm{u}+\partial_{x} \mathrm{v}^{\mathrm{eq}}=-\partial_{x}\left(\mathrm{v}^{\star}-\mathrm{v}^{\mathrm{eq}}\right)+\frac{1}{2} \Delta t\left[-\partial_{t t} \mathrm{u}+\lambda^{2} \partial_{x x} \mathrm{u}\right]+O\left(\Delta t^{2}\right) .
$$

The derivation of Eq. (10) over $t$ gives

$$
-\partial_{t t} \mathrm{u}+\lambda^{2} \partial_{x x} \mathrm{u}=\partial_{x} \theta+O(\Delta t)
$$

so replacing $\mathrm{v}^{\star}-\mathrm{v}^{\text {eq }}$ by its expression (12) yields

$$
\partial_{t} \mathrm{u}+\partial_{x} \mathrm{v}^{\mathrm{eq}}=\Delta t \sigma \partial_{x} \theta+O\left(\Delta t^{2}\right) .
$$

As $\mathrm{v}^{\mathrm{eq}}$ is a function of $\mathrm{u}, \mathrm{v}^{\mathrm{eq}}=\varphi(\mathrm{u})$, we have

$$
\theta=\left[\lambda^{2}-\left(\varphi^{\prime}(\mathrm{u})\right)^{2}\right] \partial_{x} \mathrm{u}+O(\Delta t),
$$

and we obtain the second-order macroscopic equation

$$
\partial_{t} \mathrm{u}+\partial_{x} \varphi(\mathrm{u})=\Delta t \sigma \partial_{x}\left(\left(\lambda^{2}-\left(\varphi^{\prime}(\mathrm{u})\right)^{2}\right) \partial_{x} \mathrm{u}\right)+O\left(\Delta t^{2}\right) .
$$




\section{Acknowledgement}

The numerical illustrations were performed by using a Python code LBMpy developed in the Laboratory of Mathematics (University Paris Sud). This code was optimized (parallelization, subroutines in Cython, interfacing with Cuda) by Loïc Gouarin (CNRS, University Paris Sud, Orsay).

\section{References}

[1] J. Wang, D. Wang, P. Lallemand, L.-S. Luo, Lattice Boltzmann simulations of thermal convective flows in two dimensions, Comput. Math. Appl. 65 (2013) 262-286.

[2] P. J. Dellar, Lattice and discrete Boltzmann equations for fully compressible flow, in: K.-J. Bathe (Ed.), Computational Fluid and Solid Mechanics 2005, Proceedings of The Third MIT Conference on Computational Fluid and Solid Mechanics, Elsevier, Amsterdam, 2005, pp. 632-635.

[3] P. J. Dellar, Two routes from the boltzmann equation to compressible flow of polyatomic gases, Progress in CFD 8 (2008) 94-96.

[4] F. Chen, A. Xu, G. Zhang, Y. Li, Prandtl number effects in MRT lattice Boltzmann models for shocked and unshocked compressible fluids, Theoretical and applied mechanics letters 1 (2011) 052004(4pp).

[5] F. Chen, A. Xu, G. Zhang, Y. Li, Multiple-relaxation-time lattice Boltzmann model for compressible fluids, Physics Letters A 375 (2011) 2129-2139.

[6] A. Xu, G. Zhang, Y. Gan, F. Chen, X. Yu, Lattice Boltzmann modeling and simulation of compressible flows, Frontiers of Physics 7 (2012) 582-600.

[7] S. Jin, Z. Xin, The relaxation schemes for systems of conservation laws in arbitrary space dimensions, Comm. Pure Appl. Math 48 (1995) 235-277.

[8] D. Aregba-Driollet, R. Natalini, Convergence of relaxation schemes for conservation laws, Applicable Analysis 61 (1996) 163-193.

[9] R. Natalini, B. Hanouzet, Weakly coupled systems of quasilinear hyperbolic equations, Diff. Integral Eq 9 (1993) 1279-1292.

[10] F. Berthelin, F. Bouchut, Relaxation to Isentropic Gas Dynamics for a BGK System with Single Kinetic Entropy, Meth. and Appl. of Analysis 9 (2002) 313-327.

[11] F. Berthelin, F. Bouchut, Kinetic invariant domains and relaxation limit from a BGK model to isentropic gas dynamics, Asymptot. Anal. 31 (2002) 153-176.

[12] M. Pelanti, F. Bouchut, A Relaxation Method for Modeling Two-Phase Shallow Granular Flows, in: Proceedings of Symposia in Applied Mathematics, volume 67(2), "Hyperbolic problems. Theory, numerics and applications", Proceedings of the 12th international conference on hyperbolic problems, Univ. Maryland, College Park, 2008, 2009, pp. 835-844.

[13] M. Junk, A Finite Difference Interpretation of the Lattice Boltzmann Method, Numer. Methods Partial Differ. Equations 17 (2001) 383-402.

[14] D. d'Humière, Generalized lattice-boltzmann equations, in: Rarefied Gas Dynamics: Theory and simulation, volume 159, AIAA Progress in astronomics and aeronautics, 1992, pp. 450-458.

[15] F. Dubois, Une introduction au schéma de boltzmann sur réseau, ESAIM Proc. 18 (2007) 181-215.

[16] F. Dubois, Equivalent partial differential equations of a lattice Boltzmann scheme, Computers and Mathematics with Applications. 55 (2008) 1441-1449.

[17] S. N. Kružkov, First order quasilinear equations in several independant variables, Math. USSR Sb. 10 (1970).

[18] M. Bouzidi, M. Firdaouss, P. Lallemand, Momentum transfer of a Boltzmann-lattice fluid with boundaries, Physics of Fluids 13 (2001) 3452-3459. 